\title{
Beam dynamics design studies of a superconducting radioactive ion beam postaccelerator
}

\author{
M. A. Fraser, ${ }^{1,2,3, *}$ R. M. Jones, ${ }^{1,2}$ and M. Pasini ${ }^{3,4}$ \\ ${ }^{1}$ University of Manchester, School of Physics and Astronomy, Oxford Road, Manchester M13 9PL, United Kingdom \\ ${ }^{2}$ The Cockcroft Institute, Daresbury, Warrington, Cheshire WA4 4AD, United Kingdom \\ ${ }^{3}$ CERN, CH-1211, Geneva 23, Switzerland \\ ${ }^{4}$ Instituut voor Kern-en Stralingsfysica, K.U. Leuven, Celestijnenlaan 200D, B-3001 Leuven, Belgium
}

(Received 6 October 2010; published 17 February 2011)

\begin{abstract}
The HIE-ISOLDE project at CERN proposes a superconducting upgrade to increase the energy range and quality of the radioactive ion beams produced at ISOLDE, which are currently postaccelerated by the normal conducting radioactive ion beam experiment linac. The specification and design choices for the HIE-ISOLDE linac are outlined along with a comprehensive beam dynamics study undertaken to understand and mitigate the sources of beam emittance dilution. The dominant cause of transverse emittance growth was attributed to the coupling between the transverse and longitudinal motions through the phase dependence of the rf defocusing force in the accelerating cavities. A parametric resonance induced by the coupling was observed and its excitation surveyed as a function of transverse phase advance using numerical simulations and analytic models to understand and avoid the regions of transverse beam instability. Other sources of emittance growth were studied and where necessary ameliorated, including the beam steering force in the quarter-wave resonator and the asymmetry of the rf defocusing forces in the solenoid focusing channel. A racetrack shaped beam port aperture was shown to improve the symmetry of the fields in the high- $\beta$ quarter-wave resonator and reduce the loss of acceptance under the offset used to compensate the steering force. The methods used to compensate the beam steering are described and an optimization routine written to minimize the steering effect when all cavities of a given family are offset by the same amount, taking into account the different velocity profiles across the range of mass-to-charge states accepted. The assumptions made in the routine were shown to be adequate and the results well correlated with the beam quality simulated in multiparticle beam dynamics simulations. The specification of the design tolerances is outlined based on studies of the sensitivity of the beam to misalignment and errors, with particular emphasis on the phase and amplitude stability required for the independently phased quarter-wave resonators.
\end{abstract}

DOI: 10.1103/PhysRevSTAB.14.020102

\section{INTRODUCTION TO REX AND HIE-ISOLDE}

The radioactive ion beam experiment (REX) at ISOLDE has provided the nuclear physics community with a wide range of postaccelerated radioactive ion beams (RIBs) since 2001 and can currently deliver beams with energies up to $2.8 \mathrm{MeV} / \mathrm{u}$ using a normal conducting linear accelerator shown in black in Fig. 4 [1,2]. A Penning trap and electron beam ion source (EBIS) allows the postacceleration of heavy nuclides with mass-to-charge states $(A / q)$ below 4.5 at low emittance. The HIE-ISOLDE project aims to increase the quality, intensity, and energy of RIBs produced at CERN through a major upgrade to the ISOLDE facility [3]. We present the research and development activities focused on the beam dynamics design of the upgrade of the postaccelerator.

\footnotetext{
*Corresponding author. mfraser@cern.ch
}

PACS numbers: 29.27.-a, 41.85.-p, 29.20.Ej, 29.38.-c

\section{DESIGN CHOICES FOR THE HIE-ISOLDE LINAC}

The design of the HIE-ISOLDE linac was driven by the request for a fully energy variable machine capable of boosting RIBs with $A / q$ lighter than 4.5 to at least $10 \mathrm{MeV} / \mathrm{u}$ in the existing ISOLDE experimental hall. This demands a real-estate gradient of more than $2 \mathrm{MV} / \mathrm{m}$ in the $22 \mathrm{~m}$ of space available after the REX radio frequency quadrupole (REX-RFQ). The aim was to achieve the target energy with full operational flexibility, while maintaining beam quality. In 2006 an International Advisory Board reviewed both normal conducting and superconducting (SC) upgrade proposals and supported an approach based on independently phased superconducting quarter-wave resonators (QWRs) [4]. The superconducting option efficiently provides the high gradients which facilitate the use of short accelerating structures in the space available at ISOLDE, hence allowing continuous energy variation without degradation of beam quality. Through experience at RIB facilities such as at TRIUMF, ANL, and LNL, QWRs were sighted early in the design 
stage as a reliable and robust accelerating structure on which to base the HIE-ISOLDE design. By boosting the beam after the RFQ to $1.2 \mathrm{MeV} / \mathrm{u}$ using the existing $5 \mathrm{MV}$ interdigital $\mathrm{H}$-mode (IH) structure, only two families and a total of 32 QWRs are needed to reach the design energy at the nominal accelerating gradient of $6 \mathrm{MV} / \mathrm{m}$ per cavity. The SC linac could also be rephased to operate in a decelerating mode opening up a new energy regime at ISOLDE below $1.2 \mathrm{MeV} / \mathrm{u}$, which would be of particular interest to the nuclear astrophysics community. The HIEISOLDE project is pursuing niobium sputtered copper QWRs and profiting from the experience at CERN of sputtered accelerating cavities, albeit in a different velocity regime and geometry. Therefore, the design of the cavity geometry must remain as simple as possible to present a surface capable of being sputtered. The final major choice influencing the beam dynamics design was the selection of solenoids for the transverse focusing channel. The most important benefit of using solenoids instead of quadrupoles at HIE-ISOLDE is that they can be more easily integrated alongside the cavities inside the cryomodules, reducing the overall length of the machine, especially at low energy, and increasing the packing factor. As a result the longitudinal acceptance is increased. Solenoids have a higher tolerance to mismatch and a higher acceptance to beams of multiple charge states, permitting the use of stripping foils should higher energies be desired [5]. In addition, the single tuning knob makes them easier to operate when scaling to accelerate radioactive beam.

\section{FIRST-ORDER BEAM DYNAMICS DESIGN STUDIES}

The first-order beam dynamics simulations were carried out using the linear accelerator numerical analysis (LANA) code, which was used to design and commission the ISAC DTL and ISAC II machines at TRIUMF [6]. The transverse particle distribution is generated with a 4D Waterbag distribution for the transverse phase space and a separate 2D Waterbag distribution is generated for the longitudinal phase space, where $\epsilon_{T \text {,tot }}=6 \epsilon_{T, \text { rms }}$ and $\epsilon_{L, \text { tot }}=4 \epsilon_{L, \text { rms }}$. All the elements of the HIE-ISOLDE linac were modeled in the hard-edge approximation, have axial symmetry about the beam axis, and the transit time factor has no radial dependence. The motions are strongly coupled. In the transverse plane the motions in the horizontal and vertical planes are coupled by the solenoid and therefore in order to avoid emittance exchange the initial distribution of particles in phase space was made axially symmetric.

\section{A. Specification of the accelerating structures}

The specification demands that the SC cavities provide 39.6 MV of accelerating potential within approximately $18 \mathrm{~m}$, taking into account the existing normal conducting infrastructure and assuming a $1 \mathrm{~m}$ matching section for the SC linac. At a packing factor of 50\%, a synchronous phase $\left(\phi_{s}\right)$ of $-20^{\circ}$, and an average transit time factor $(T)$ of 0.8 , one arrives within the nominal accelerating gradient $\left(E_{0}\right)$ of $6 \mathrm{MV} / \mathrm{m}$. A solution was found for the lattice by applying the first-order expression for the energy gain of a two gap resonator [7],

$$
\frac{\Delta W}{A}=\frac{q}{A} E_{0} L_{a} T_{2 \mathrm{gap}}(\beta) \cos \phi_{s}
$$

where

$$
T_{\text {2gap }}(\beta)=\frac{\sin \frac{\pi g}{\beta \lambda}}{\frac{\pi g}{\beta \lambda}} \sin \frac{\pi \beta_{g}}{2 \beta} .
$$

$\beta_{g}$ is the geometric reduced velocity of the cavity, $g$ is the width of the gaps whose centers are separated by $\beta_{g} \lambda / 2$ and $E_{0}$ is assumed constant within the gaps. In the first instance the gap width was assumed to be zero. The number of cavity families was minimized to just two $\left(\beta_{g}=6.3\right.$ and $\left.10.3 \%\right)$, comprising 12 and 20 cavities, respectively, in order to boost the beam energy from 1.2 to over $10 \mathrm{MeV} / \mathrm{u}$. The active length occupied by each cavity $\left(L_{a}\right)$ was approximated by $\beta_{g} \lambda$ and the total active length for the above solution was kept below $9 \mathrm{~m}$, in accordance with a 50\% packing factor. The choice of such a high synchronous phase reflects the demanding energy gain specification but also helps the transverse beam stability. The flexibility of the beam energy, which can be varied by switching off cavities, is illustrated in Fig. 1(a) for the upper and lower bounds of the $A / q$ acceptance, determined by the limitations of the normal conducting linac. Also shown is the low energy section of the SC linac rephased in a decelerating mode. In this approximation the beam can be decelerated down to $0.5 \mathrm{MeV} / \mathrm{u}$, limited by the zero in the first-order transit time factor. The two cavity designs were developed in the Microwave Studio (MWS) environment. An in-depth description of the rf design can be found in [8,9]. In order to commence first-order beam dynamics studies in LANA the transit time factors for the two cavities were calculated numerically from the field maps extracted from MWS and fitted with the analytic result that was presented in Eq. (1). At first order, the change of ion velocity inside the cavity is neglected. The transit time factors were fitted as is shown alongside the accelerating fields in Fig. 2. The fitted parameters were used to generate Fig. 1(b). One can see a drop in acceleration efficiency from Fig. 1(a) to Fig. 1(b) which arises from the finite gap width of the realistic cavity, however, enough tolerance was left for the design energy to be met within the available space at a packing factor of $50 \%$ and nominal gradient. The optimum velocity at which the acceleration efficiency is maximized also shifts away from the geometric velocity to higher values as a result of the finite gap width. The lightest ions can be accelerated close to $17 \mathrm{MeV} / \mathrm{u}$. 


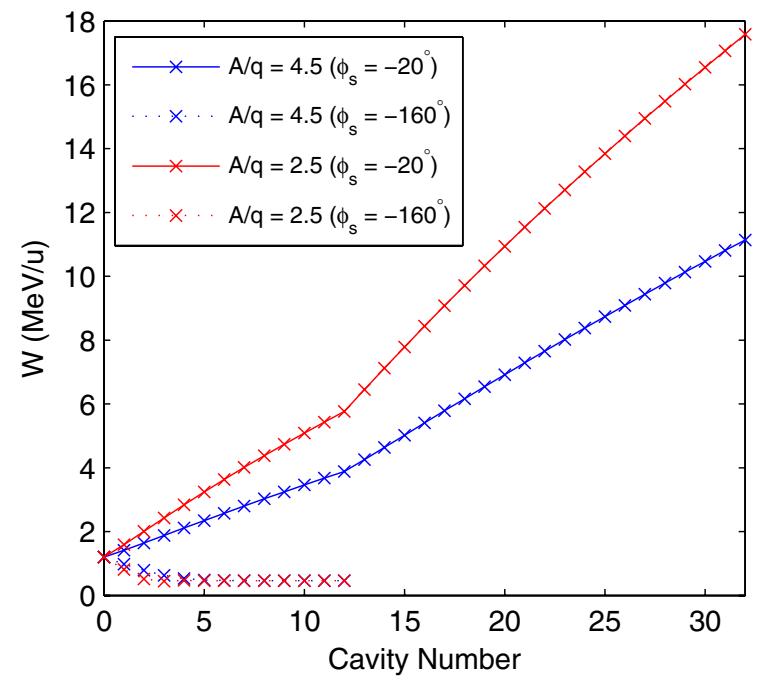

(a)In the thin gap approximation, $g=0$.

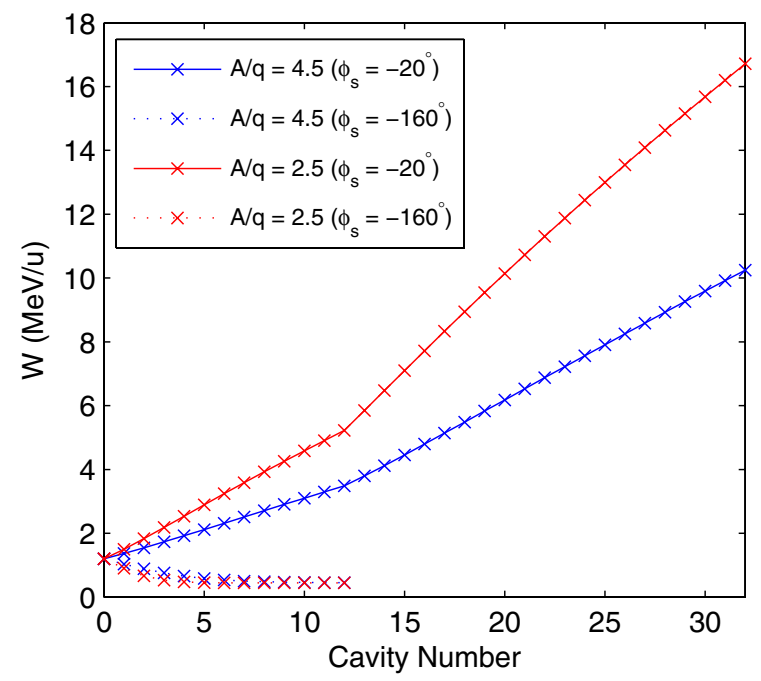

(b) Using the realistic accelerating field calculated in MWS.

FIG. 1. A first-order calculation of the energy profile along the linac for $A / q=2.5$ and 4.5 in both acceleration and deceleration modes.

\section{B. Lattice design choices}

The first choice was to divide the linac by grouping the two types of cavity families together. The low energy section contains two cryomodules, each housing six low- $\beta$ cavities and two solenoids, and the high energy section contains four cryomodules, each housing five high- $\beta$ cavities and one solenoid, as shown in Fig. 3. The modularity of the linac facilitates a staged installation and allows for the management of manpower and the use of available funding throughout the upgrade. It is foreseen to install two high energy cryomodules after the REX linac in a first stage, before augmenting the linac with more high energy cryomodules and replacing all the existing normal conducting accelerators after the IH structure with the low energy section. The first stage will increase the energy to

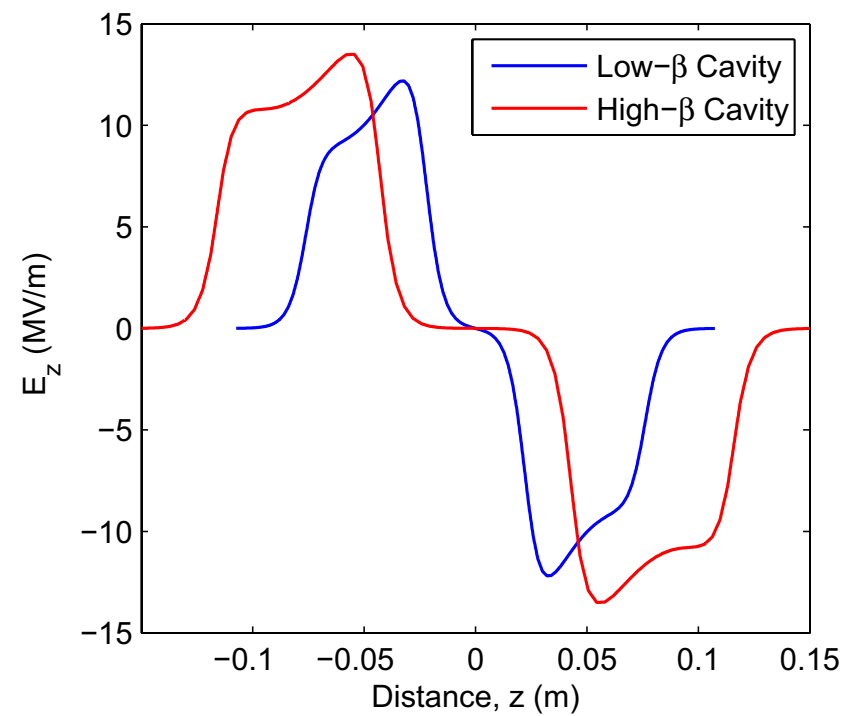

(a)The accelerating field profiles.

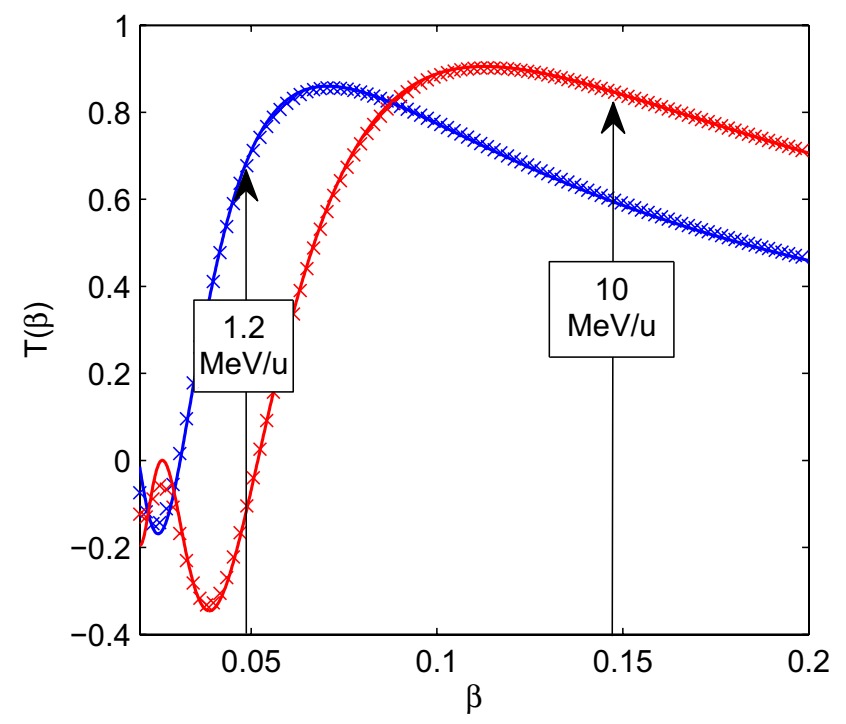

(b)The transit time factors calculated numerically and fitted with the analytic result shown in Eq. 2.

FIG. 2. The accelerating properties of the low $-\beta\left(\beta_{g}=6.3 \%\right)$ and high- $\beta\left(\beta_{g}=10.3 \%\right)$ cavities.

$5.5 \mathrm{MeV} / \mathrm{u}$ before the design energy of $10 \mathrm{MeV} / \mathrm{u}$ is reached along with full energy flexibility on completion of the upgrade and installation of the low energy section, as shown in Fig. 4. A critical design parameter is the packing factor which affects not only the overall length of the linac but also the longitudinal acceptance and its optimization was a primary objective of the lattice design. The independent phasing of the cavities is exploited by incorporating the longitudinal matching section after the IH structure into the first cryomodule of the low energy section, removing the need for a dedicated buncher and saving valuable space in the experimental hall. The in-line matching is achieved using the first cavity as a buncher and separating it from the other cavities with a drift region containing a solenoid. 


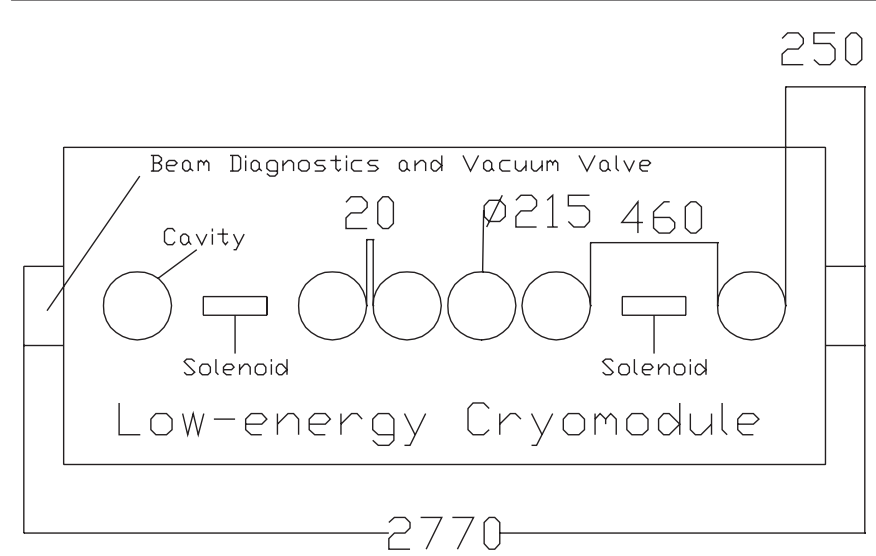

(a)

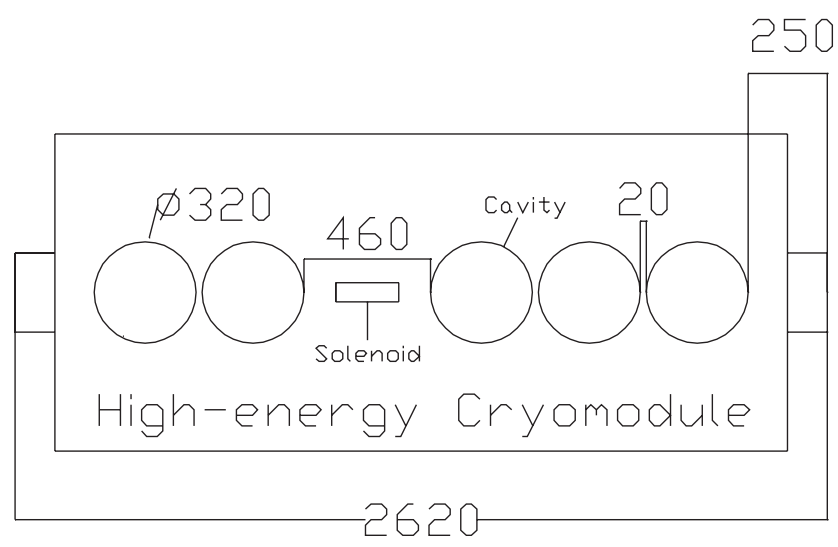

(b)

FIG. 3. The layout of the low (a) and high (b) energy cryomodules. Distances are in units of $\mathrm{mm}$.

A synchronous phase of just $-40^{\circ}$ is adequate to capture the beam by minimizing the phase spread in the second cavity. The last cavity in the low energy cryomodule is also separated with a solenoid, making itself and the fifth cavity useful for completing the longitudinal phase space gymnastics early in the acceleration. In order to match the beam transversely between the two sections of the SC linac, an irregular lattice is employed in the high energy cryomodule with just two cavities before the solenoid and three after. This reduces the beam divergence across the transition region between the last solenoid in the low energy section and the first in the high energy section. No adjustment of the synchronous phases is needed to match the beam into the high energy section in both the intermediate and complete upgrade scenarios. To standardize the lattice design, all the high energy cryomodules are kept irregular, the second low energy cryomodule is identical to the first, and the size of the low and high energy cryomodules kept similar. The effect of the packing factor on the longitudinal acceptance is shown in Fig. 5 with different distances between the cryomodules. In the three cases: (a), (b) and (c), the packing factor is $62 \%, 56 \%$, and $47 \%$ and the total linac length is $14.5,16.0$, and $19.0 \mathrm{~m}$, respectively. Only a small increase in acceptance is achieved by reducing the intercryomodule distance below $50 \mathrm{~cm}$ because of the space already taken up by the solenoids inside the cryomodules. The designated lattice spacing of $46 \mathrm{~cm}$ given for each solenoid ensures the magnetic field is less than $50 \mathrm{mT}$ at the adjacent cavity when fully powered; the solenoid design is focused on driving down the fringe field.

\section{Suppression of longitudinal-transverse coupling}

The models of the solenoid and cavity in the first-order simulations are axially symmetric and therefore the radial phase space coordinates $\left(r, r^{\prime}=\frac{d r}{d z}\right)$ are used. Although the low beam intensities at ISOLDE negate the effect of spacecharge induced instabilities, permitting nonadiabatic acceleration, there is strong coupling of the transverse and longitudinal motions in the cavities which must be controlled to avoid resonant growth of the transverse beam emittance. The predominant source of coupling arises from the phase dependence of the rf defocusing force which acts as a parametric perturbation on the transverse beam dynamics. The other source of coupling results from the radial dependence of the transit time factor. Starting from the uncoupled equations of motion, the coupled equations of motion can be derived by approximating the radial dependence of the accelerating field with $E \approx E_{0}(1+$ $K r^{2} / 2$ ) and expanding the phase for small oscillations

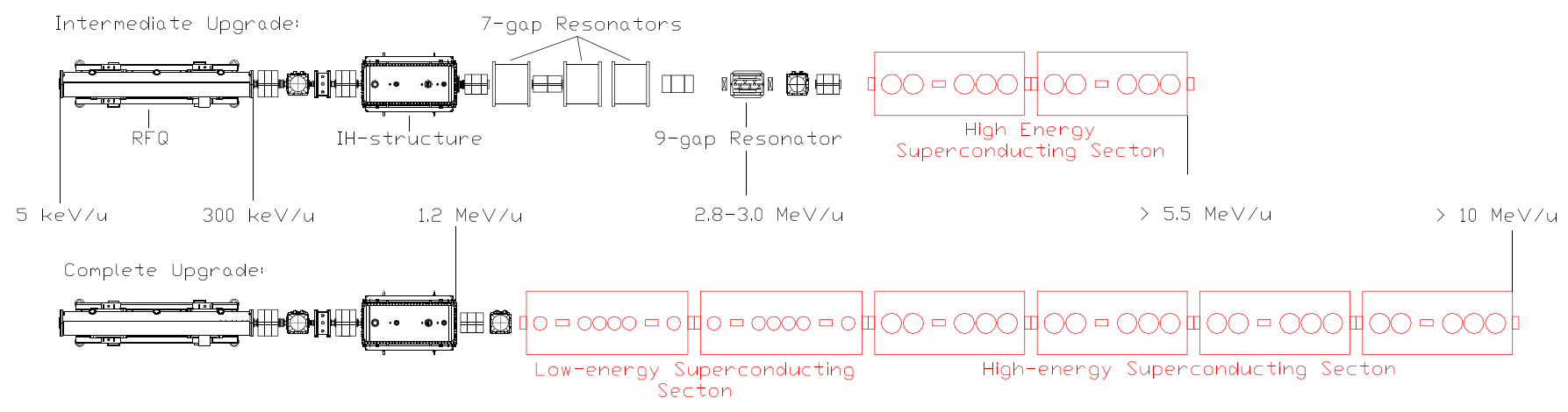

FIG. 4. The lattice design of the HIE-ISOLDE linac. 


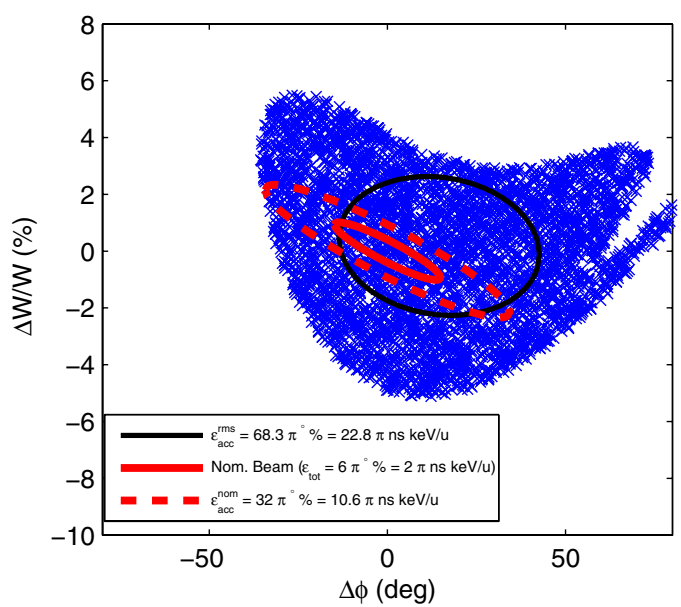

(a)Packing factor $=62 \%$ : inter-cryomodule dist. $=25 \mathrm{~cm}$.

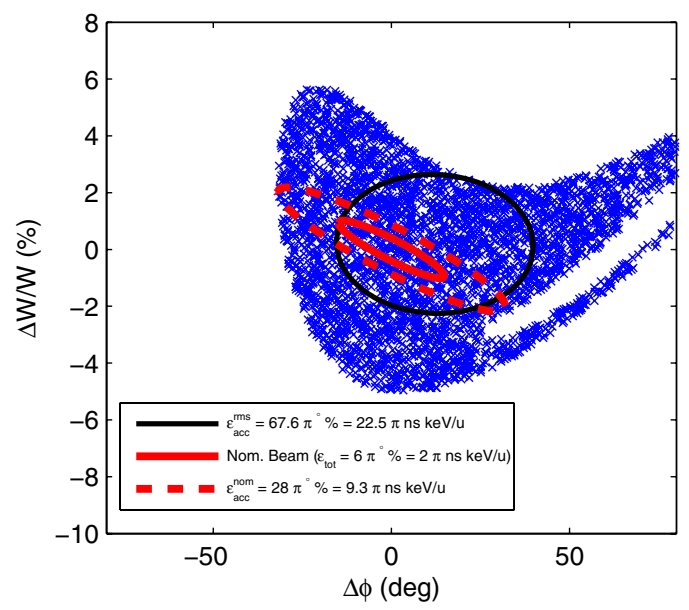

(b)Packing factor $=56 \%$ : inter-cryomodule dist. $=50 \mathrm{~cm}$.

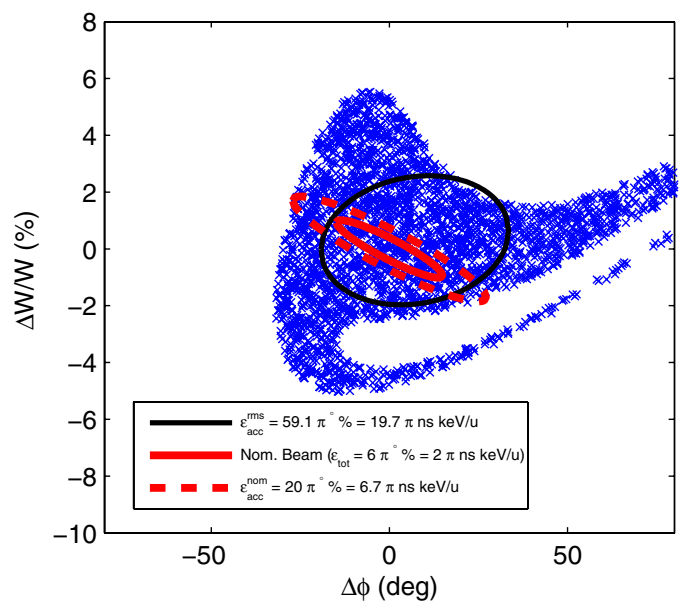

(c)Packing factor $=47 \%$ : inter-cryomodule dist. $=100$ $\mathrm{cm}$.

FIG. 5. The longitudinal acceptance in the complete upgrade with different intercryomodule distances. The nominal beam at injection is presented and the acceptance quantified by maximizing the emittance of the nominal beam as well as by fitting an rms ellipse to the accepted particle distribution. about the synchronous particle such that $\phi=\Delta \phi+\phi_{s}$, where $r, \Delta \phi \ll 1$, and $K=\frac{2 \pi}{\beta_{s} \lambda}$. The transverse and longitudinal equations of motion with coupling terms up to first order in $\Delta \phi$ are shown in Eqs. (3) and (4):

$$
\begin{gathered}
\frac{1}{\beta_{s} \gamma_{s}} \frac{d}{d z}\left[\beta_{s} \gamma_{s} \frac{d r}{d z}\right]+k_{T}^{2} r=k_{L_{s}}^{2} \frac{\Delta \phi \cot \phi_{s}}{2} r \\
\frac{1}{\beta_{s}^{3} \gamma_{s}^{3}} \frac{d}{d z}\left[\beta_{s}^{3} \gamma_{s}^{3} \frac{d \Delta \phi}{d z}\right]+k_{L_{s}}^{2} \Delta \phi \\
=-k_{L_{s}}^{2}\left(\frac{\pi r}{\beta_{s} \gamma_{s} \lambda}\right)^{2}\left[\cot \phi_{s}+\Delta \phi\right] .
\end{gathered}
$$

$k_{L_{s}}$ is the longitudinal oscillation wave number of the synchronous particle,

$$
k_{L_{s}}^{2}=\frac{2 \pi q E_{0} \sin \left(-\phi_{s}\right)}{m c^{2} \lambda \beta_{s}^{3} \gamma_{s}^{3}},
$$

and $k_{T}$ is the transverse oscillation wave number of the smoothed solenoid focusing channel including the rf defocusing force for the synchronous particle,

$$
k_{T}^{2}=\left(\frac{q B_{0}}{2 m c \beta_{s} \gamma_{s}}\right)^{2}-\frac{k_{L_{s}}^{2}}{2} .
$$

All symbols have their usual notation where $\lambda$ is the $\mathrm{rf}$ wavelength, $m c \beta_{s} \gamma_{s}$ is the momentum of the synchronous particle, $q$ is the charge of the particle, and $B_{0}$ is the smoothed value of the solenoid magnetic field. The longitudinal equation of motion couples at second order to the transverse motion through the radial dependence of the transit time factor and is thus neglected in the first-order studies. The longitudinal equation of motion remains uncoupled, i.e., for $r \ll 1$,

$$
\frac{1}{\beta_{s}^{3} \gamma_{s}^{3}} \frac{d}{d z}\left[\beta_{s}^{3} \gamma_{s}^{3} \frac{d \Delta \phi}{d z}\right]+k_{L_{s}}^{2} \Delta \phi=0 .
$$

The phase spread of a bunch mismatches the focusing channel for every nonsynchronous particle within the bunch causing an increase of the transverse beam emittance, which must be carefully controlled to avoid the buildup of beam halo. Although the beam halo is not a specific concern for safety or machine stability because of the very low beam power $(\sim \mathrm{mW})$, it is for the experiments. The dilution of transverse emittance is driven by the size of the longitudinal emittance. The effects of zerocurrent coupling in low energy ion linear accelerators (below about $10 \mathrm{MeV} / \mathrm{u}$ ) are described analytically by Gluckstern (see, for example, [10-12]), and summarized in [13]. Importantly, it was shown that as a result of the coupling there exist regions of parameter space within which the transverse motion is unstable. In particular, if the smoothed wave numbers satisfy the condition,

$$
k_{T}=\frac{j}{2} k_{L} \quad j=1,2,3 \ldots
$$


the transverse equation of motion is resonantly unstable. The conditions for transverse stability are derived by assuming adiabatic changes in the beam parameters during acceleration in the smooth approximation, which cannot be strictly applied to the HIE-ISOLDE linac. However, many of Gluckstern's conclusions describe the general behaviors observed in the numerical simulations we present.

In order to simplify the analysis, the linac was split into its two sections and the transverse emittance growth in both the low and high energy sections analyzed separately. The longitudinal beam parameters were fixed in both sections equivalent to those used for acceleration to the design energy with $A / q=4.5$. The growth of the rms and $99 \%$ normalized transverse emittances as a function of the strength of the solenoid focusing channel was simulated with total longitudinal emittances of 2 and $4 \pi \mathrm{keV} / \mathrm{uns}$, the former being the design emittance. The results are presented in Fig. 6 by parametrizing the strength of the focusing channel with the transverse phase advance per focusing period $\left(\mu_{T}\right)$, where the period length is the center to center distance of adjacent solenoids. The effect of the longitudinal emittance driving the transverse emittance growth is clearly demonstrated. The coupling is suppressed with stronger focusing channels and as a consequence at higher phase advances the transverse emittance growth depends only very weakly on the longitudinal emittance. At phase advances close to $90^{\circ}$ per focusing period the emittance growth is minimized. In this study the intercryomodule distance was $80 \mathrm{~cm}$ and the total length of the linac close to $18 \mathrm{~m}$. The strength of the transverse-longitudinal coupling is reduced as the packing factor and the longitudinal acceptance are increased because the bunch can be constrained tighter in its phase extent. To investigate this effect the intercryomodule distance was halved and the study repeated. The overall emittance growth is reduced and the minimum of the emittance growth is observed to shift to lower phase advances because of the reduction in the longitudinal phase advance. To date, the longitudinal emittance of the REX linac, which will act as the booster for the upgrade, has not been accurately measured and therefore the specification of the solenoid is based upon a strong focusing channel of $90^{\circ}$ per period to decouple the effect of the longitudinal emittance and ensure the emittance growth is minimized even if the longitudinal emittance is found to be larger than the design value. This specification demands an integrated solenoid field of up to $16.2 \mathrm{~T}^{2} \mathrm{~m}$ for the heaviest ions at $10 \mathrm{MeV} / \mathrm{u}$.

In the high energy section the variation of the longitudinal phase advance along the linac can be linearized and the emittance growth factor calculated analytically using the first-order result derived by Gluckstern,

$$
\Delta \epsilon_{T(z \rightarrow \infty)}^{1 \text { st order }} \approx \frac{k_{L_{s}}^{2}}{4 k_{T}\left|2 k_{T}-k_{L_{s}}\right|} \cot \left(-\phi_{s}\right) \Delta \phi_{0},
$$

where the emittance growth can be twice as large before settling down to this limit in a long accelerating channel.

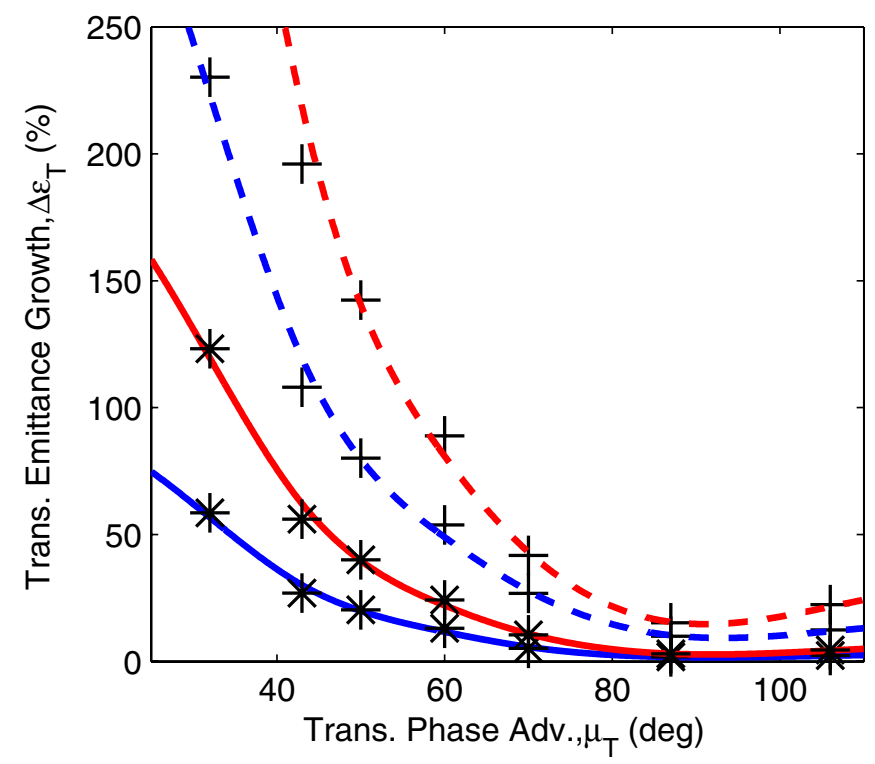

(a)Low energy section.

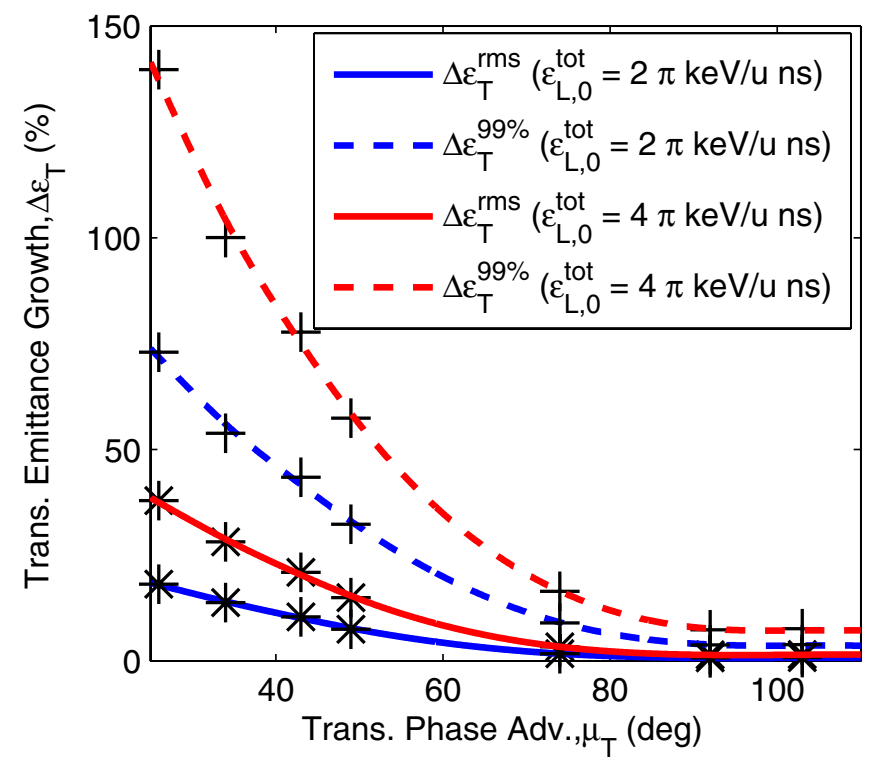

(b)High energy section.

FIG. 6. The transverse emittance growth as a function of transverse phase advance per focusing period with an intercryomodule distance of $80 \mathrm{~cm}$.

The application of this prediction to the high energy section of the HIE-ISOLDE linac is shown in Fig. 7, along with the second-order prediction, $\Delta \epsilon_{T(z \rightarrow \infty)}^{2 \text { nd order }}$ [12]. The emittance growth compares well with the numerical simulation at higher phase advances but the resonance peak itself is not resolved numerically. The likely cause of the discrepancy is that at low phase advance with only four periods the beam has not relaxed to the limit made in the analytic estimate. In addition, the longitudinal phase advance varies with acceleration which introduces a range of unstable oscillation frequencies and a broadening of the 


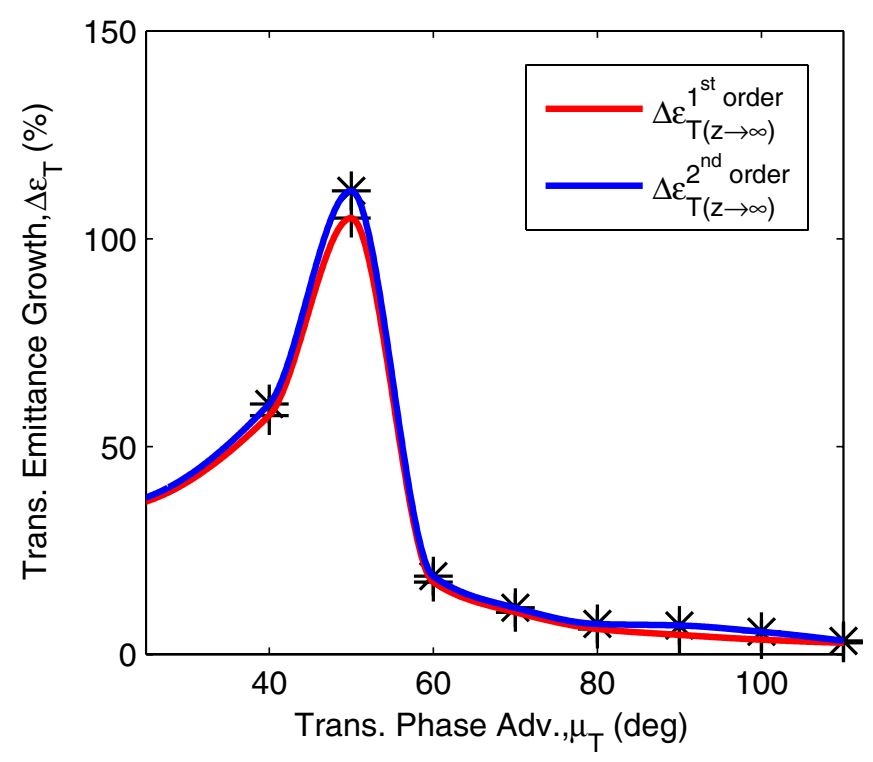

FIG. 7. The first-order analytic prediction of the emittance growth in the high energy section calculated using Eq. (9) assuming a longitudinal emittance of $2 \pi \mathrm{keV} / \mathrm{u}$ ns. Alongside, the second-order prediction is plotted.

resonance. Although the second-order parametric resonance is not observed within the range of phase advances investigated in the numerical simulations of the high energy section, there is evidence in Fig. 6(a) of the secondorder resonance being excited in the low energy section. The instability conditions change as a function of $A / q$. Although the resonant conditions lie at higher phase advances for lower $A / q$ beams, it was shown that as a result of the increased energy gain of the lighter beams the focusing channel does not require retuning to phase advances higher than $90^{\circ}$ per period [14]. The sensitivity of the emittance growth to the beam parameters at low energy, where the phase spread is largest and the rf defocusing force strongest, is shown in Fig. 8 for an intercryomodule distance of $80 \mathrm{~cm}$. This study includes both sections of the linac as would be present in the complete upgrade. The strength of the focusing channel in the low energy section was varied and matched into a focusing channel of $60^{\circ}$ per period in the high energy section. A region of instability is crossed in the low energy section that causes a resonant growth in the transverse emittance. In contrast to the simulations of the separate sections of the linac, the extra length allows the emittance growth to relax at low phase advances and the resonance peak is resolved. There is also evidence for the excitation of the second-order instability at higher phase advances.

\section{Beam dynamics simulations of the nominal case}

The nominal parameters for a beam of $A / q=4.5$ accelerated to $10 \mathrm{MeV} / \mathrm{u}$ at a transverse phase advance per period close to $90^{\circ}$ are presented in Fig. 9. The total design emittances at injection are $0.3 \pi \mathrm{mm} \mathrm{mrad}$ and

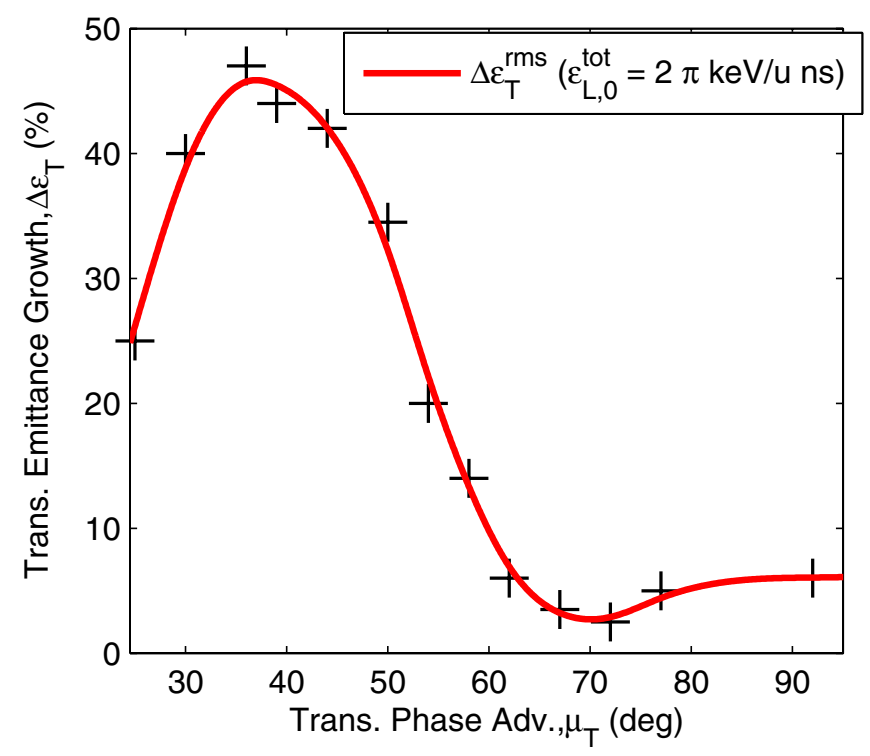

FIG. 8. The resonant growth in transverse emittance at output as a function of the focusing strength in the low energy section.

$2.0 \pi \mathrm{keV} / \mathrm{u}$ ns and no particles were lost in the simulation. The output longitudinal parameters can be manipulated by changing the phase and amplitude of the final cavity and the energy spread can be rotated to less than $0.25 \%$ at $10 \mathrm{MeV} / \mathrm{u}$. The rms and $99 \%$ transverse emittance growth is kept to 1.0 and $6.0 \%$, respectively, at ejection after an initial excitation in the low energy section is suppressed. The longitudinal emittance growth that arises just after injection results from the large phase spread of the beam in the first cavity. The design of the longitudinal phase space gymnastics in the low energy lattice allows this nonlinear effect to be largely reduced before the beam enters into the high energy section. The absence of a dedicated matching section across the transition region between the low and high energy sections does not significantly affect the beam quality. Different order resonant conditions are met transiently all along the linac but the variation of the longitudinal phase advance prevents an instability growing.

In order to match the beam into a focusing channel of $90^{\circ}$ per period in the high energy section, the beam has to be prepared before reaching the transition region by lowering the phase advance in the low energy section. The beam envelope function is then larger on entry to the transition region and diverges less across it, enabling stronger focusing channels to be matched in the high energy section. On the evidence of Fig. 6, one would expect a larger growth of emittance as a consequence of lowering the phase advance in the low energy section instead of the high energy section. The emittance growth is not significant if the phase advance in the second low energy cryomodule is lowered and the strength of the solenoids in the first cryomodule kept at $90^{\circ}$ per period. The phase advance was lowered as far as $50^{\circ}$ in the period 

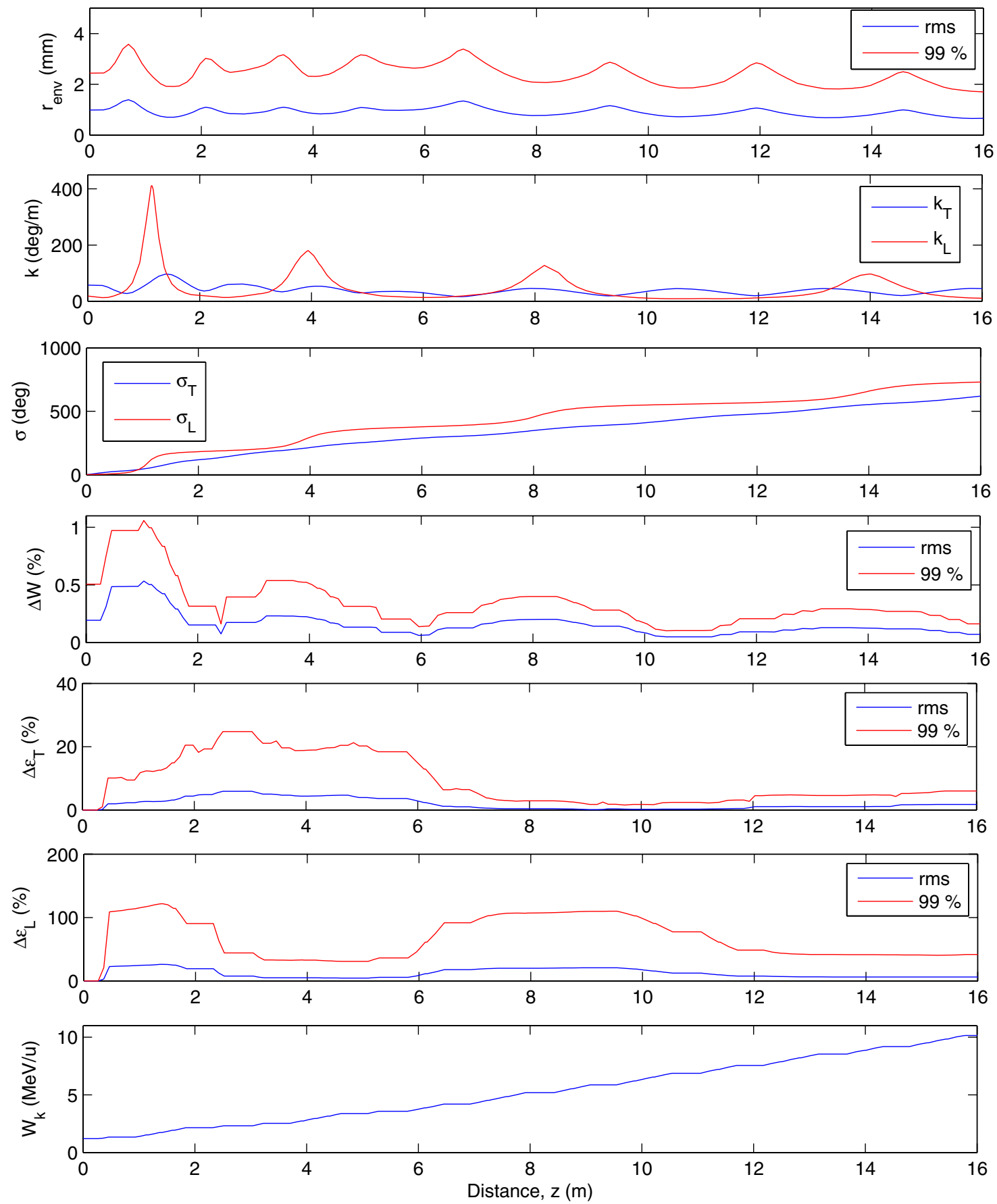

FIG. 9. The nominal beam parameters simulated at first order using the LANA code: $A / q=4.5$ to $10 \mathrm{MeV} / \mathrm{u}$.

used for matching without significant degradation of the beam quality, in contradiction to what Fig. 6(a) suggests. One can therefore conclude that the initial beam parameters are critical and that lowering the transverse phase advance at injection seriously affects the emittance growth along the linac, making the design of the matching section after the normal conducting linac critical. A study of the beam quality was also made in which the phase advance was kept at $90^{\circ}$ per period throughout the low energy section. In this case the strongest focusing channel that could be matched across the transition region was $60^{\circ}$ per period in the high energy section. The beam emittance was 
not increased in the low energy section but as a result of the lower phase advance in the high energy section the emittance at output was approximately $10 \%$ larger, in accordance with Fig. 6(b).

\section{BEAM DYNAMICS DESIGN STUDIES WITH REALISTIC FIELDS}

The study was extended to include the realistic fields of the QWRs and solenoids allowing other sources of emittance growth to be investigated. The multiparticle beam dynamics simulations were carried out using the TRACK code which solves the equations of motion numerically by tracking each particle through the field maps of the linac structures [15]. The particle distribution was simulated using a 6D Waterbag and the rms emittance scaled to make comparisons between LANA and TRACK simulations. The compensation of the beam steering force is the main topic of discussion in this section with a specific focus on the high energy section and the high- $\beta$ cavity.

\section{A. The beam steering force in the QWRs}

The geometry of the high- $\beta$ cavity in the region of the beam ports was described by two parameters. The first parameter $\left(y_{0}\right)$ represents the height of the center of the beam port above the end of the straight part of internal conductor. The second parameter $(\Delta)$ represents the vertical offset of the noses with respect to the center of the beam port such that the beam port is asymmetrically positioned on the nose when $\Delta$ is nonzero, as shown in Fig. 10. A third parameter $(\delta)$ was used to describe the vertical offset of the beam axis, defined by the magnetic axis of the aligned solenoids, with respect to the center of the beam port. $\delta$ represents the injection offset of the beam inside the aperture and is equivalently an offset of the entire cavity with respect to the beam axis. The definition of these parameters is presented in Fig. 11.

The electromagnetic fields on the beam port axis are characteristic of QWRs at this frequency [16]. The electric and magnetic contributions to the steering force for a particle of $A / q=4.5$ entering on axis at a beam port height of $40 \mathrm{~mm}$ and at the nominal synchronous phase of $-20^{\circ}$ are shown in Fig. 12 as a function of velocity. The calculations were done by tracking a single particle representing the beam centroid through the electromagnetic

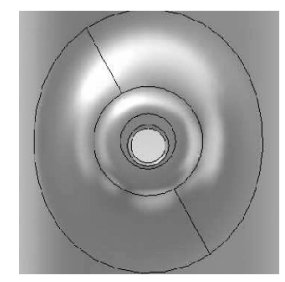

(a) $\Delta>0$

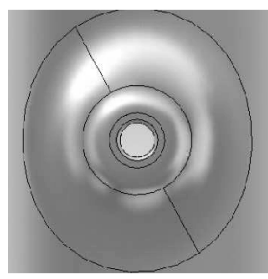

(b) $\Delta=0$

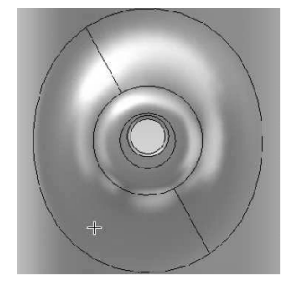

(c) $\Delta<0$
FIG. 10. The position of the center of the noses relative to the center of the beam port aperture parametrized by $\Delta$.
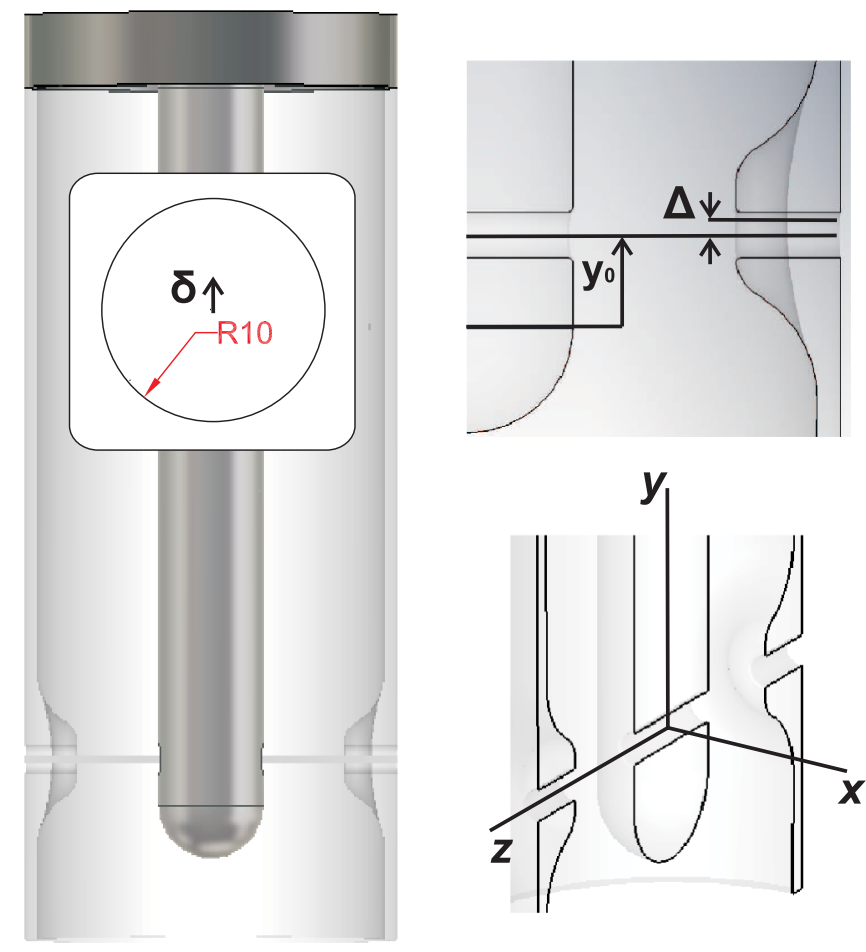

FIG. 11. The geometry of the nominal (NOM) high- $\beta$ cavity. The beam offset $(\delta)$ inside the nominal aperture is shown inset on the left and a positive value of $\Delta$ shows a vertical offset of the noses with respect to the center of the beam port on the top right.

field map of the resonator and are compared to two simple analytic approximations: the leading-order Fourier approximation [16] and the square-wave approximation [17]. The steering is caused mainly by the magnetic field circulating about the inner conductor but at velocities below the geometric velocity the electric dipole field can have a significant effect. Figures 12(c) and 12(d) show how the steering force is compensated by offsetting the entry position of the beam centroid vertically above the axis of the beam port by $\delta=2.4 \mathrm{~mm}$. The offset makes the beam centroid sample the rf defocusing fields produced by the aperture [16]. An analytic expression can be derived to help understand the basic properties of the steering force and its mitigation. The steering angle imparted in a QWR can be expressed in general as

$$
\begin{aligned}
\Delta y^{\prime}= & -\underbrace{\frac{q\left|E_{y, a v}\left(y_{0}\right)\right| L_{a}}{A \gamma u c^{2} \beta^{2}} P(\beta) \sin \phi_{s}}_{\text {electric term }} \\
& +\underbrace{\frac{q \mu_{0}\left|H_{x, a v}\left(y_{0}\right)\right| L_{a}}{A \gamma u c \beta} Q(\beta) \sin \phi_{s}}_{\text {magnetic term }} \\
& -\underbrace{\delta \frac{\pi q\left|E_{z, a v}\left(y_{0}\right)\right| L_{a}}{A \lambda \gamma u c^{2} \beta^{3}} R(\beta) \sin \phi_{s}}_{\text {rf defocusing term }}
\end{aligned}
$$




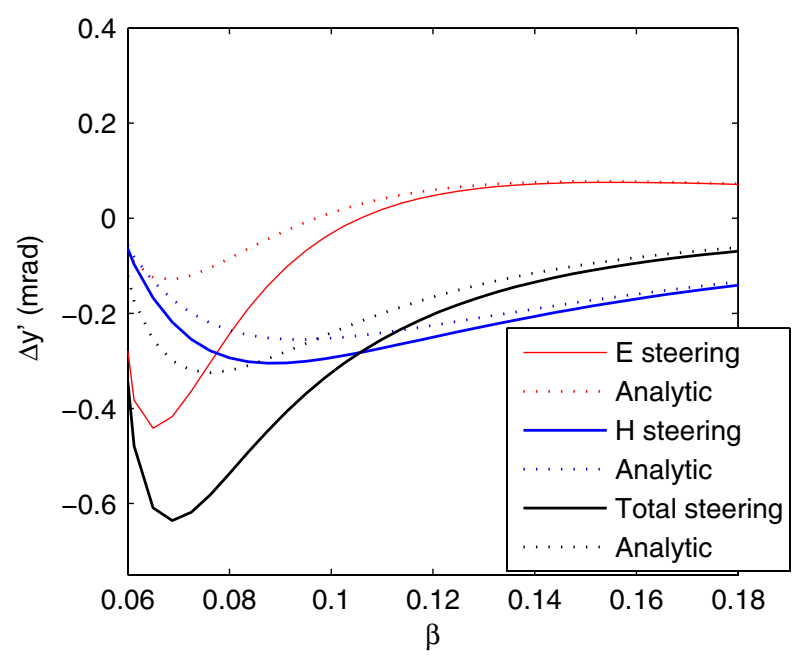

(a) Uncompensated $(\delta=0 \mathrm{~mm})$ with analytic leading-order Fourier approximation.

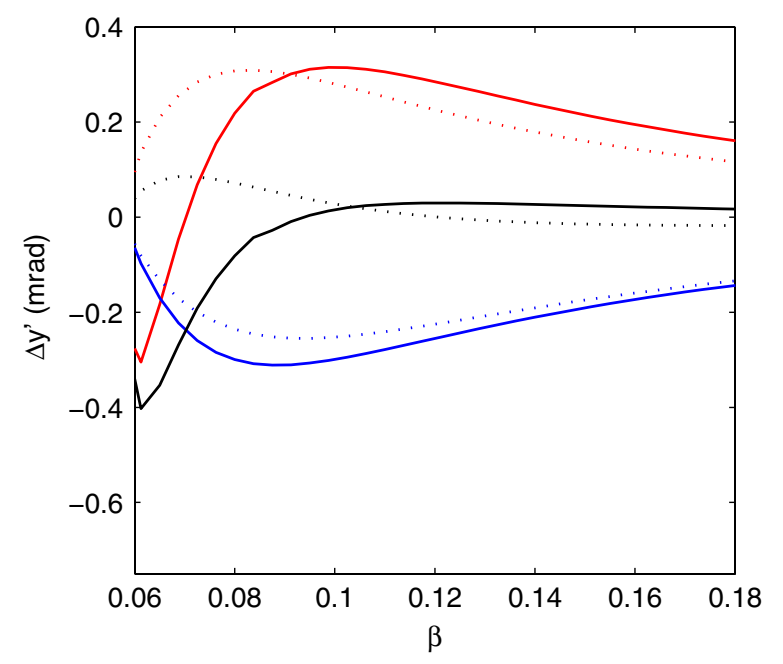

(c)Compensated $(\delta=2.4 \mathrm{~mm})$ with analytic leading-order Fourier approximation.

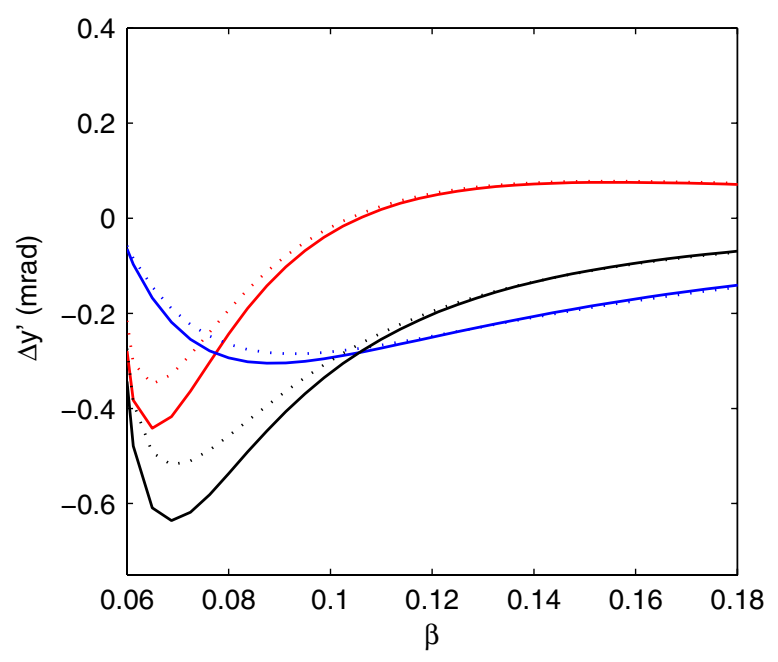

(b) Uncompensated $(\delta=0 \mathrm{~mm})$ with analytic square-wave approximation.

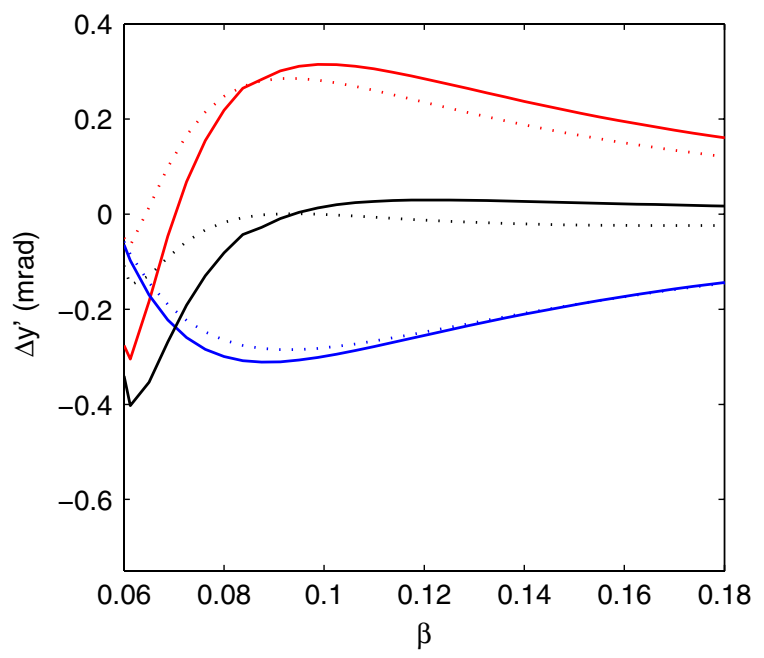

(d)Compensated $(\delta=2.4 \mathrm{~mm})$ with analytic square-wave approximation.

FIG. 12. The steering effect in the high- $\beta$ cavity for $A / q=4.5$ operating at a synchronous phase of $-20^{\circ}$.

where $u$ is an atomic mass unit and $\mu_{0}$ the permeability of free space. The expression assumes that the field components remain constant over the offset $\delta$ and that the particle deflection and change in velocity can be neglected inside the cavity. The field components are stated in terms of the average field value in each gap. The factors $P(\beta), Q(\beta)$, and $R(\beta)$ are form factors that describe the dynamics of the interaction between a charged particle and the electromagnetic fields, which depend on the profile of the fields along the axis. In principle, the factors can be calculated numerically from the realistic field profiles and used to separate the dependence on velocity and phase in an analogous way to the transit time factor. All three components of the steering force have the same phase dependence $\left(\propto \sin \phi_{s}\right)$, which allows the transverse-longitudinal coupling and the resulting emittance growth to be suppressed, something that would not be possible with external steering elements. Although the leading-order Fourier approximation gives reasonable agreement with numerical calculations, the steering effect can be better described by representing the fields as constant in the gaps as in the square-wave approximation. This allows the gap size to be varied independently of the geometric velocity of the structure and the extra fitting parameter improves the description of the transit time factor. The simple leadingorder Fourier approximation is useful when studying low- $\beta$ resonators where the drift tube length is short. The magnetic steering is described well by both models. The deterioration in the quality of the analytic description of the compensated steering effect, see Figs. 12(c) and 12(d), 
arises from the description of the rf defocusing force which assumes axial symmetry about the offset beam axis. Nonetheless, the analytic results explain why although the electric dipole force in each gap cancels at the geometric velocity, at low velocity it dominates and increases the overall steering by acting in the same direction as the magnetic force. The $1 / \beta^{3}$ dependence of the rf defocusing force provides the modification to the electric dipole force required to cancel the electric and magnetic forces over a wide velocity range, especially at low velocity. The quality of the compensation depends very much on the various contributions from $\left|H_{x, \text { av }}\right|,\left|E_{y, \text { av }}\right|$, and $\left|E_{z, \text { av }}\right|$, which can be optimized as a function of $y_{0}$ and $\Delta$. The steering effect has a strong phase and velocity dependence, and is inversely proportional to $A / q$; therefore the optimum parameters for compensation depend on the mass-to-charge state of the beam accelerated because the linac is independently phased and the velocity profile flexible.

\section{B. Optimization of beam steering compensation}

It is not possible to select a single beam port position and cavity offset such that the steering is exactly canceled in all the cavities along the linac due to the different velocity dependence of the electric and magnetic components of the steering force. One could imagine a mechanical solution able to manipulate the offset of each cavity individually, tunable to the mass-to-charge state and velocity profile of the beam; however, a single offset for all cavities within a given family is adequate to control the emittance growth.

A single-particle tracking routine was developed to find the optimum cavity geometry over the range of beams to be accelerated. Focusing on the intermediate upgrade in normal operation, the beam centroid was tracked in the high- $\beta$ cavity and the injection velocity looped to track the velocity profile in all the cavities along the high energy section. Assuming the correction of position and divergence after each cavity, the steering in each cavity was calculated and the quality of compensation assessed by taking the rms deviation from zero $\left(\sigma_{\Delta y^{\prime}}\right)$ of the ensemble of 20 kicks as a function of $y_{0}, \Delta$, and $\delta$. A survey of $\sigma_{\Delta y^{\prime}}$ in the high energy section operating with $\phi_{s}=-20^{\circ}$ is presented in Fig. 13 for beams of $A / q=4.5$ and 2.5 injected at 2.8 and $3.0 \mathrm{MeV} / \mathrm{u}$ respectively. The height of the beam port was varied and the beam port kept centered on the nose, i.e. $\Delta=0 \mathrm{~mm}$. The key results are collected in Table I. The correlation between $\sigma_{\Delta y^{\prime}}$ and the simulated emittance growth in TRACK multiparticle simulations is presented in Fig. 14 and justifies the assumptions and use of the figure of merit in the cavity optimization routine. For the case of $A / q=4.5$, the beam steering is minimized at a cavity offset of about $2.5 \mathrm{~mm}$ for beam port heights between 40 and $60 \mathrm{~mm}$. $\left|E_{y, \text { av }}\right|$ increases rapidly close to the end of the internal conductor, causing a fast degradation in the compensation at low velocity; however, the optimum value of the cavity offset decreases as a result of the increase in the

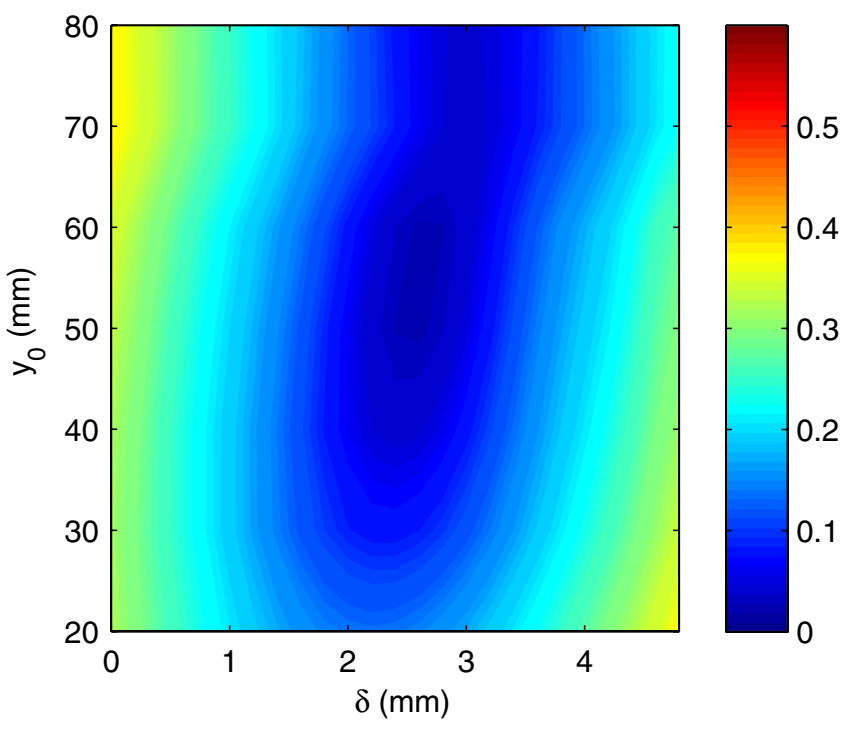

(a) $A / q=4.5$.

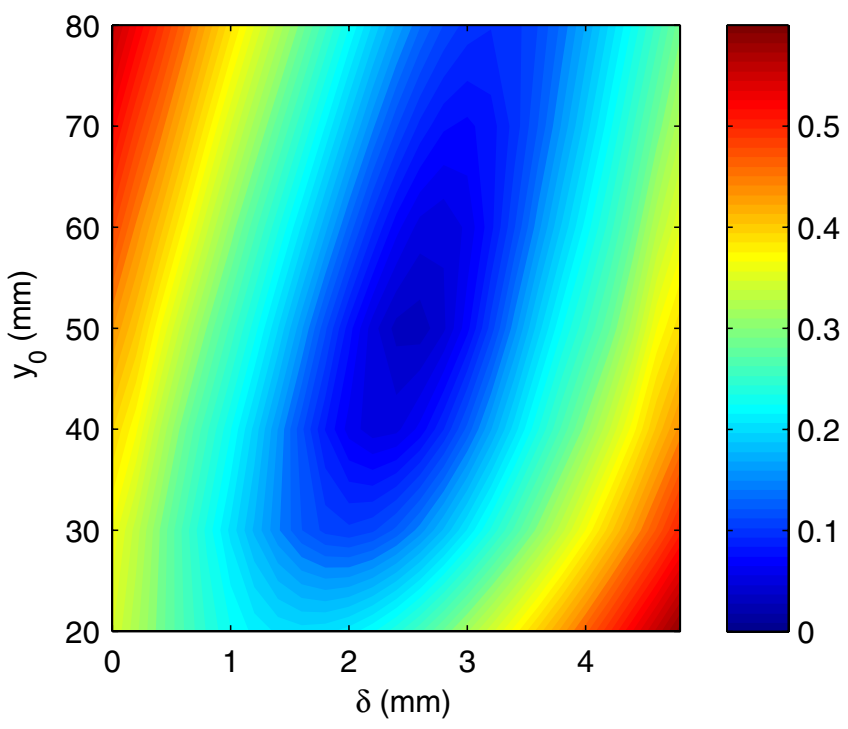

(b) $A / q=2.5$.

FIG. 13. Contours of $\sigma_{\Delta y^{\prime}}$ (mrad) in the high energy section as a function of $y_{0}$ and $\delta$, with the beam ports centered on the nose $(\Delta=0 \mathrm{~mm})$.

ratio $\left|E_{z, \text { av }}\right| /\left|H_{x, \text { av }}\right|$ and less vertical acceptance is lost on offset. The geometrical parameter $R / Q$ decreases at $6 \Omega / \mathrm{cm}(\sim 1 \% / \mathrm{cm})$ with the height of the beam port in the high- $\beta$ cavity. The values of $\delta$ required to minimize the steering effect for beams with different mass-to-charge states diverge as the beam port height moves away from the optimum height of about $50 \mathrm{~mm}$, making an effective compensation over a range of $A / q$ more difficult. A beam port height of $40 \mathrm{~mm}$ was chosen to gain a satisfactory compensation performance without significant loss in shunt impedance but also because of the proximity of the optimum values of $\delta$ for $A / q=2.5$ and 4.5 , as shown in Fig. 14. Interestingly, the higher injection energy of the lighter beam and the increased velocity it develops in the 
TABLE I. A summary of the optimization of $y_{0}$ and $\delta$ $(\Delta=0 \mathrm{~mm})$ in the high energy section.

\begin{tabular}{lccc}
\hline \hline & & $\begin{array}{c}\delta_{\text {opt }}(\mathrm{mm}) \\
y_{0}(\mathrm{~mm})\end{array}$ & $\begin{array}{c}\text { Loss of vertical } \\
\text { acceptance }(\%) \\
(A / q=4.5 / 2.5)\end{array}$ \\
\hline 20 & $5 / Q(\Omega)$ & $(A / q=4.5 / 2.5)$ & $39.2 / 29.4$ \\
30 & 564 & $2.2 / 1.6$ & $40.7 / 36.0$ \\
40 & 559 & $2.3 / 2.0$ & $42.2 / 40.7$ \\
50 & 553 & $2.6 / 2.5$ & $45.2 / 43.8$ \\
60 & 545 & $2.6 / 2.8$ & $45.2 / 48.2$ \\
70 & 539 & $2.8 / 3.0$ & $48.2 / 51.0$ \\
80 & 531 & $3.0 / 3.2$ & $51.0 / 53.8$ \\
\hline \hline
\end{tabular}

SC linac causes it to be only slightly more sensitive to steering and not by the factor $4.5 / 2.5$. The optimum offset $\delta_{\text {opt }}$ is approximately $25 \%$ of the radius of the aperture $r_{\text {ap }}$ resulting in a large loss of acceptance, see Table I. To remedy this without affecting the acceleration efficiency significantly, the aperture was elongated in the vertical direction by half a radius to form a racetrack shaped aperture, as shown in the inset of Fig. 16(a). The racetrack minimizes the loss in aperture when the cavity is offset and keeps the shunt impedance high with respect to a circular beam port of the same major radius. The racetrack was also preferred to a larger circular aperture because of the improved ratio of $\delta_{\text {opt }} / r_{\text {ap }}$, see Table II, and the improved symmetry of the rf defocusing forces, presented in Fig. 17. In addition, the low beam power at ISOLDE removes the concern of beam halo losses on the aperture causing activation or degradation of cavity performance. The same optimization routine was carried out with the racetrack aperture giving $\delta_{\text {opt }}=2.8 \mathrm{~mm}$ and only a $6 \%$ loss in acceptance in comparison to a radial aperture of $10 \mathrm{~mm}$.

The case in which $\Delta$ is nonzero was also studied such that the beam port is positioned off center on the noses. The asymmetry introduces an additional electric dipole field on axis that modifies the intrinsic electric dipole of the QWR. If the nose is shifted away from the high electric field region the $\delta$ required for compensation using the $\mathrm{rf}$ defocusing force is reduced by $10 \%$. The additional electric dipole field introduced for $\Delta=2 \mathrm{~mm}$ is shown in Fig. 15(a) alongside the results of the optimization routine for shifts of $\pm 2 \mathrm{~mm}$. The modification introduces higher harmonic terms in the Fourier expansion for $E_{y, 0}$ which results in a force with the correct phase to compensate the magnetic steering. This technique is discussed in [16] where the beam port noses are significantly modified to compensate the beam steering without the need for the beam centroid to sample to $\mathrm{rf}$ defocusing fields. Significant modifications of the cavity geometry to compensate the beam steering effect are ruled out as a compensation technique in the HIE-ISOLDE linac due to the sputtering process which will be used to fabricate the cavity. The modification is small in the high- $\beta$ cavity

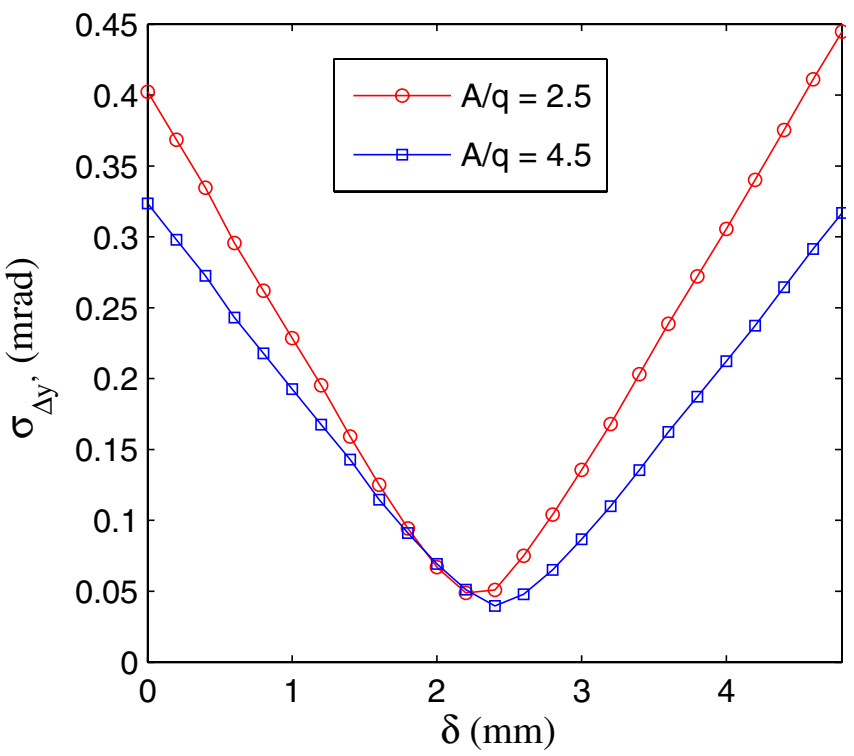

(a)Single-particle optimisation routine.

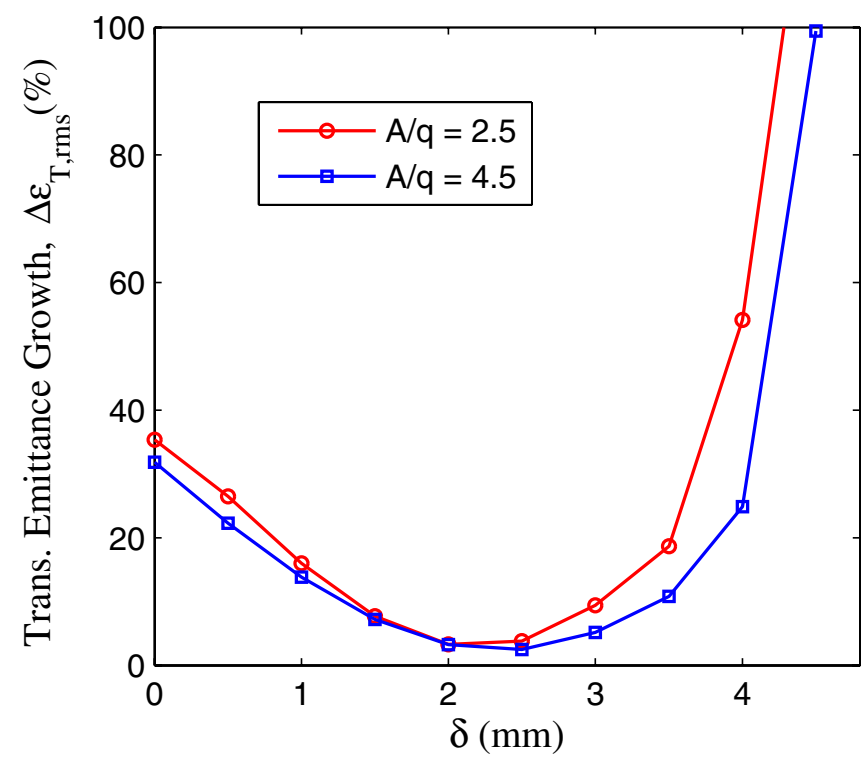

(b)Multi-particle TRACK simulations.

FIG. 14. A comparison of the single-particle optimization routine with TRACK as a function of $y_{0}$ and $\delta$, with the beam ports centered on the nose $(\Delta=0 \mathrm{~mm})$.

and easy to implement. It has a negligible effect on the asymmetry of the rf defocusing forces in the vertical plane and $\delta_{\text {opt }}$ is reduced along with the overall magnitude of the steering force. Using the racetrack aperture and $\Delta=$ $2.5 \mathrm{~mm}$ then the beam steering is optimized across the $A / q$ acceptance of the linac with $\delta=2.5 \mathrm{~mm}$.

\section{Transverse asymmetry of $\mathbf{r f}$ defocusing forces in the QWRs}

The rf defocusing fields in the high- $\beta$ cavity are intrinsically asymmetric about the beam axis. In QWRs 


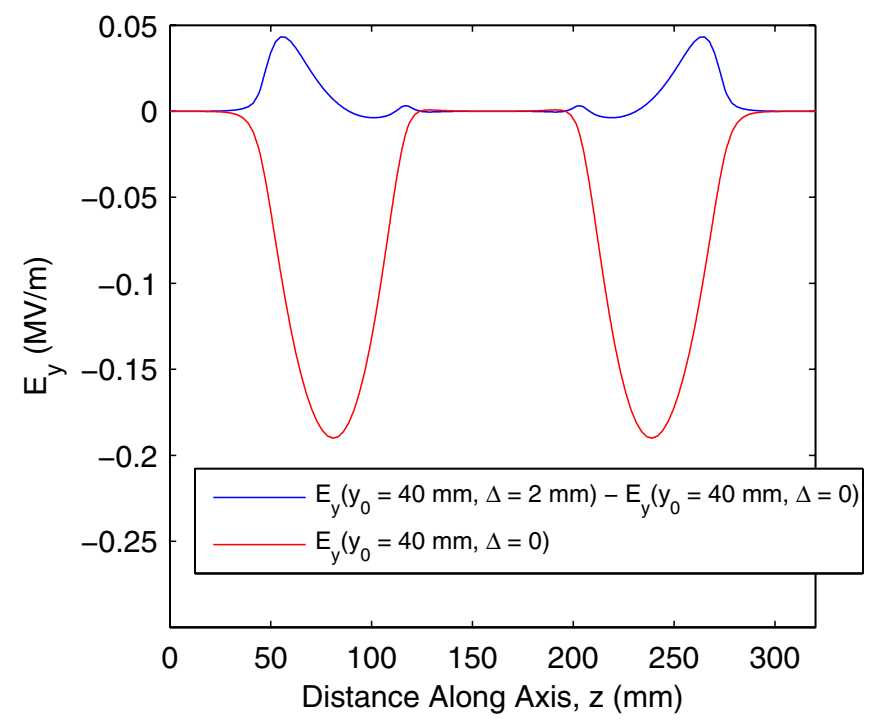

(a)The additional dipole component of electric field compared to the nominal field.

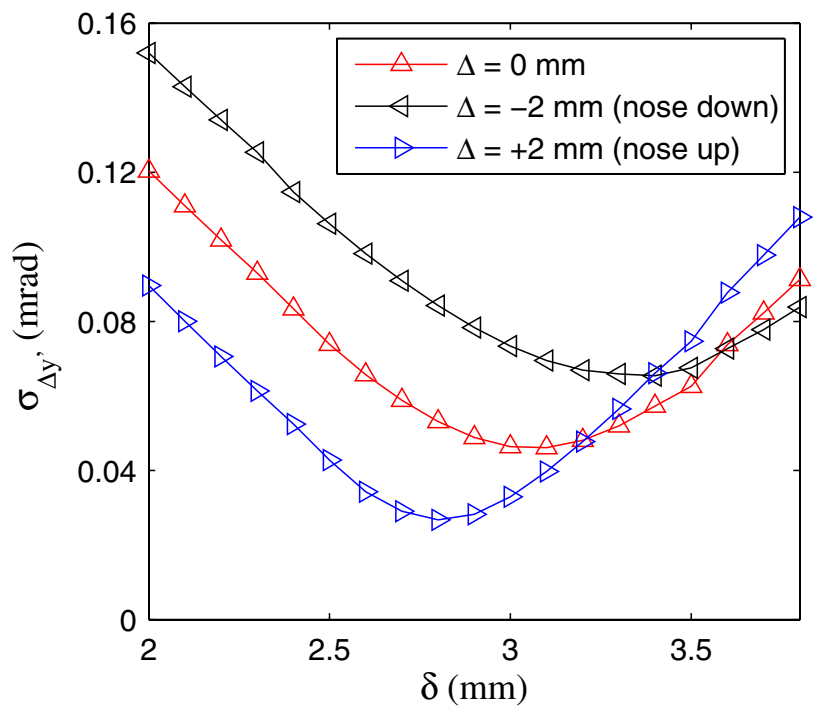

(b) $y_{0}=40 \mathrm{~mm}$ and $A / q=4.5$.

FIG. 15. The consequence of positioning the beam port $2 \mathrm{~mm}$ above and below the center of the noses $(\Delta= \pm 2 \mathrm{~mm})$.

constructed from bulk niobium it has become standard practice to improve the field symmetry by modifying the shape of the drift tube on the end of the internal resonator; however, such modifications are challenging to coat with the niobium sputtering technique. The effect of the asymmetry in the solenoid focusing channel was investigated by comparing the nominal cavity performance with two cavity variants presented in Fig. 16. The first variant is a simple racetrack shaped modification to the beam port previously discussed. The second variant has a modified drift tube achieved by tapering a flattened sphere onto the internal conductor, reflecting the shape of the beam port noses as drawn in Fig. 16(b). An extension to the bottom of the sphere is required to move the site of peak electric field
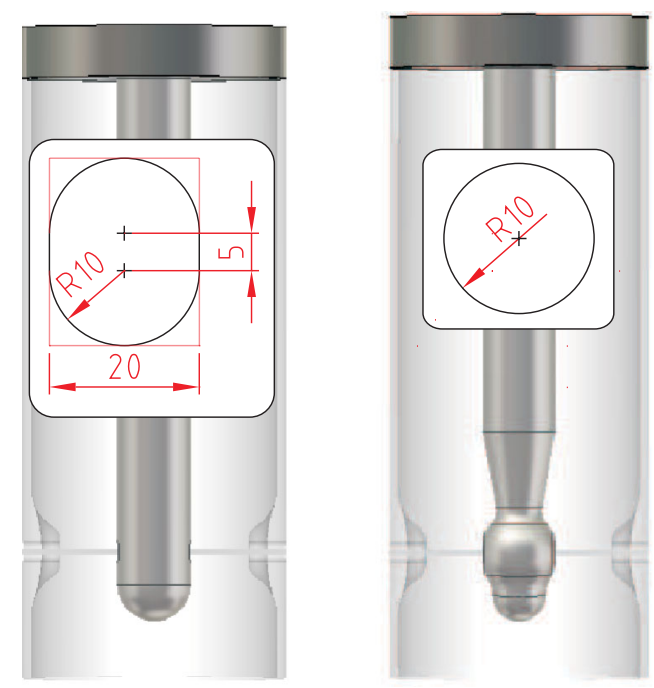

(a)Racetrack (RT) (left) and modified drift tube (MOD DT) (right).

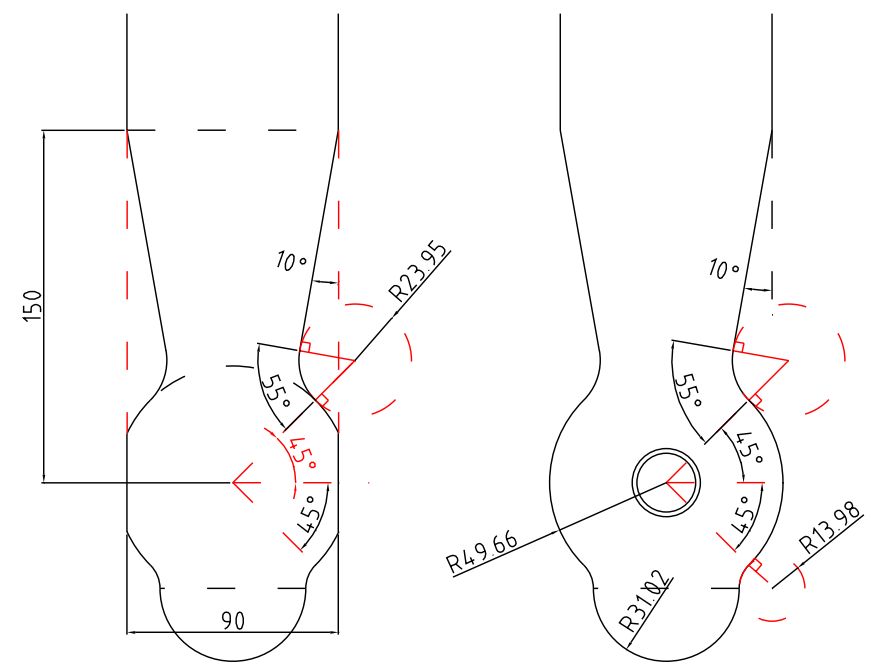

(b)The design of the modification to the internal conductor.

FIG. 16. The cavity variants. Dimensions are in $\mathrm{mm}$.

away from the beam axis such that an effective compensation of beam steering can be achieved. With the exception of the flattened faces, the modification has cylindrical symmetry about the resonator axis presenting an acceptable surface for sputtering. The rf defocusing kick was

TABLE II. A comparison between circular and racetrack beam ports.

\begin{tabular}{lcc}
\hline \hline Beam port shape & Radius (+ elongation) $(\mathrm{mm})$ & $\delta_{\text {opt }} / r_{\text {ap }}$ \\
\hline Circular & 10 & 0.25 \\
& 12.5 & 0.21 \\
Racetrack & $10(+2.5)$ & 0.22 \\
& $12.5(+6.25)$ & 0.15 \\
\hline \hline
\end{tabular}




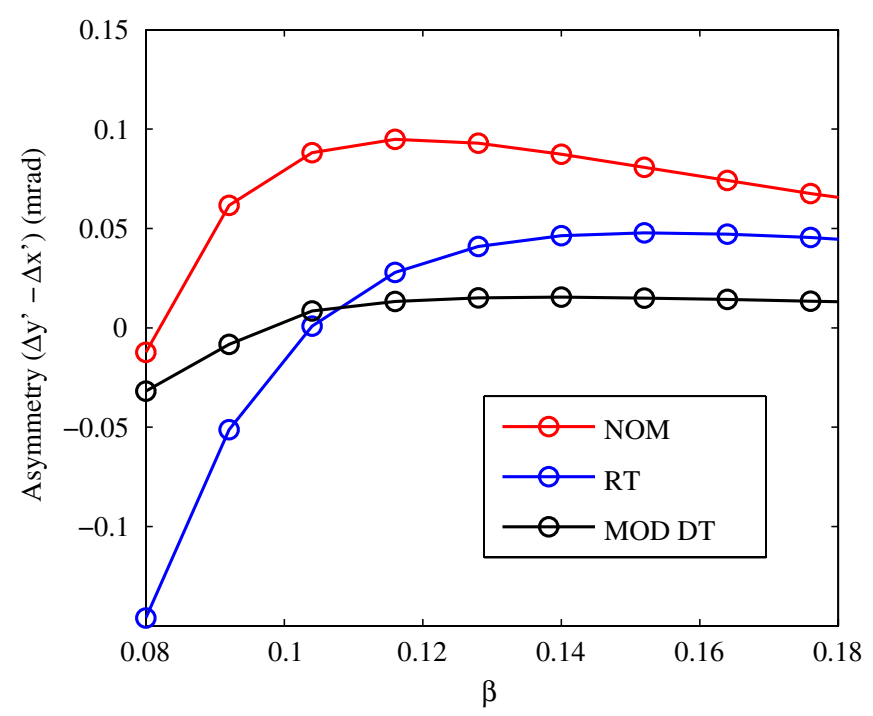

FIG. 17. The asymmetry of the rf defocusing forces in the vertical and horizontal planes for the nominal cavity and the two variants investigated.

calculated relative to the centroid at a distance of $1 \mathrm{~mm}$ in the vertical and horizontal planes by tracking a single particle in the realistic fields of the cavity variants. The results are summarized in Fig. 17. The racetrack improves the asymmetry for velocities above $\beta=0.09$ or energies of $3.7 \mathrm{MeV} / \mathrm{u}$, equivalent to the minimum injection energy to the high energy section with the low energy section installed. The modification to the internal drift tube makes the fields highly symmetric and improves the beam steering significantly at low velocity because of the reduction in the kick from the electric dipole field. The effect of the asymmetry causes different transverse phase advances in the vertical and horizontal planes which cannot both be matched with the single tuning parameter of a solenoid lens. The splitting of the horizontal and vertical beam envelopes reduces the acceptance of the linac. Moreover, the coupling of the horizontal and vertical dynamics in the solenoid and the rotation of the beam can cause an effective growth in the emittance projected onto the horizontal or vertical planes [18]. The mixing of the emittance is well controlled if the focusing channel is strong enough and the transverse-longitudinal coupling suppressed. The mixing is observed in the simulations but only at the level of a few percent by the splitting of the horizontal and vertical emittance profiles along the linac [19].

\section{Comparison between LANA and TRACK simulations}

The TRACK simulations are compared to LANA in Fig. 18 by repeating a study of the transverse emittance growth in the high energy section as a function of the strength of the focusing channel. The beam steering force is well compensated and the effect of the asymmetric rf defocusing fields and the radial dependence of the transit time factor are negligible.

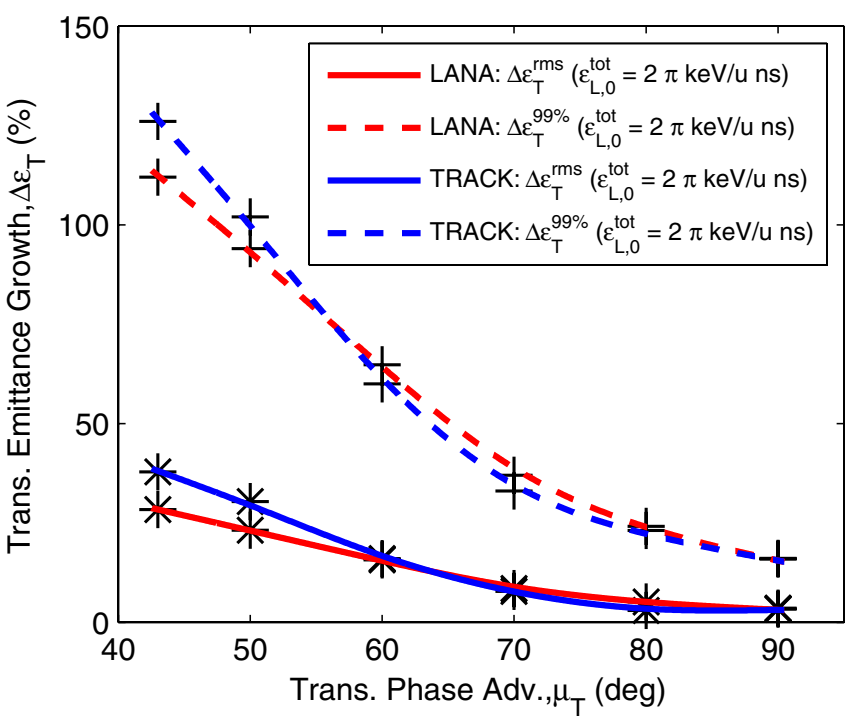

FIG. 18. A comparison of the emittance growth in the high energy section, representing the intermediate upgrade, as a function of focusing strength in the LANA and TRACK codes.

\section{E. Transverse error and misalignment study}

The lattice design was driven primarily by beam stability concerns in a machine without imperfections. The effect of the focusing strength on the transverse acceptance in the presence of misalignment was investigated using a model of the linac constructed from matrices, which parametrize the beam dynamics in the realistic cavity and solenoid fields [20]. A similar model was made to specify the error tolerances in the design of ISAC II at TRIUMF providing a quick and reliable method for systematic studies involving many error seeds [21]. The sensitivity of the beam to misalignment in the intermediate upgrade was investigated by randomly misaligning the entrance and exit positions of each element simulated through a random offset and rotation of each matrix. The position of the beam centroid is corrected using a single steerer magnet and beam position monitor (BPM) located in each intercryomodule space. The acceptance was calculated as a function of the focusing strength by superimposing the beam envelope onto the distribution of centroid trajectories in the presence of misalignment. For a given alignment tolerance the optimum working point at which the acceptance is maximized is given by a trade-off between the beam size and the beam's sensitivity to misalignment as a function of focusing strength. A survey of the optimum working points was carried out and it was found that if the linac is operated at $90^{\circ}$ phase advance per period for beam stability concerns then in order to optimize the acceptance the alignment of the solenoid axis should be controlled to within an rms deviation $\left(\sigma_{\text {sol }}\right)$ of $\pm 0.25 \mathrm{mrad}$ or a total deviation of $\pm 0.75 \mathrm{mrad}$, where the Gaussian distribution of centroid trajectories is truncated at $3 \sigma$. This corresponds to a rms alignment tolerance of $\pm 0.10 \mathrm{~mm}$ on the ends of the 


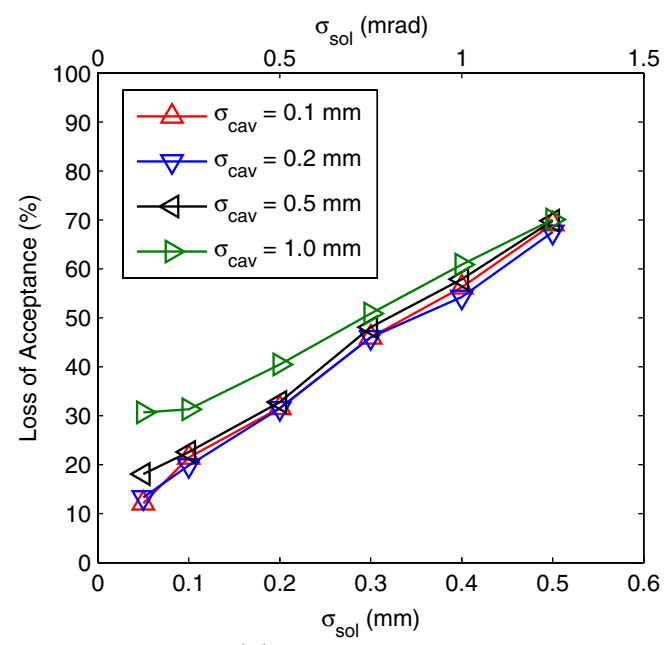

(a) $\sigma_{B P M}=0.1 \mathrm{~mm}$

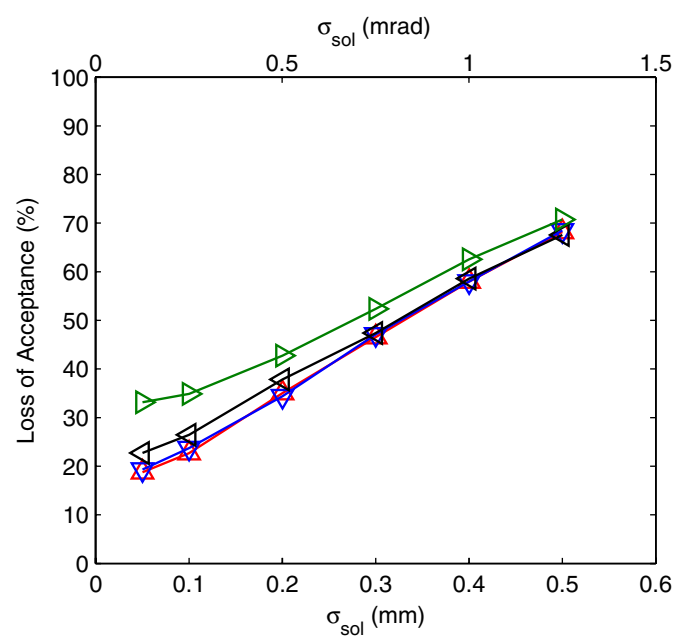

(b) $\sigma_{B P M}=0.2 \mathrm{~mm}$

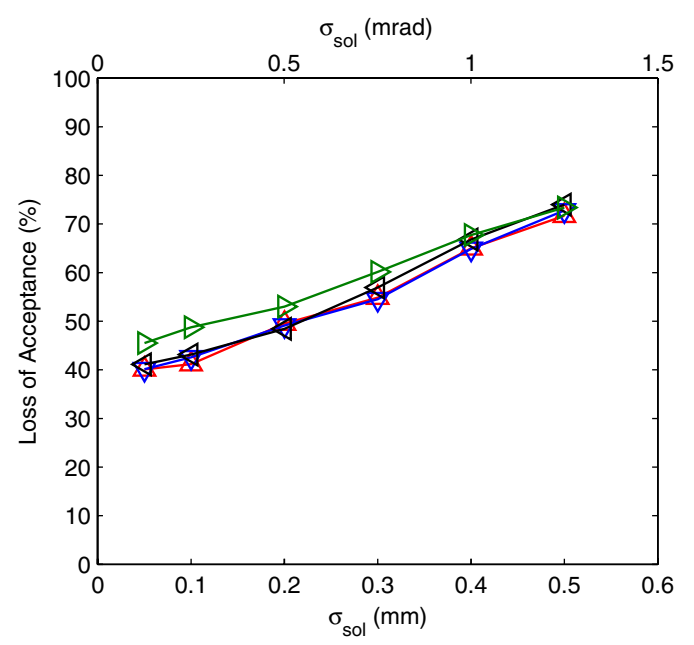

(c) $\sigma_{B P M}=0.5 \mathrm{~mm}$

FIG. 19. A survey of the loss in acceptance operating at $90^{\circ}$ phase advance per period as a function of the solenoid alignment tolerance for BPM tolerances in the range $0.1-0.5 \mathrm{~mm}$ and cavity tolerances in the range $0.1-1.0 \mathrm{~mm}$.

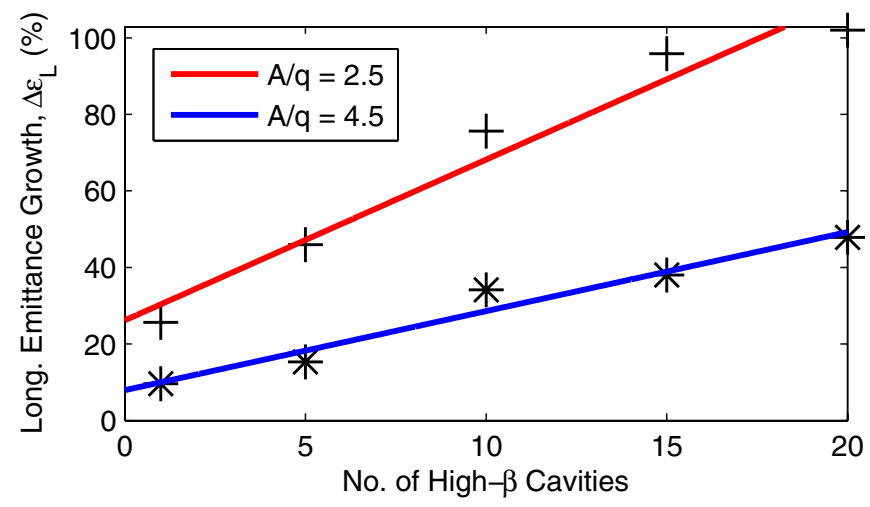

FIG. 20. The time-averaged rms emittance growth as a function of the number of high- $\beta$ cavities in the intermediate upgrade for a total jitter of $1^{\circ}$ and $1 \%$ in amplitude and phase, respectively.

solenoid with a mechanical length of $0.4 \mathrm{~m}$. Recent studies based on an optical alignment system have shown this error tolerance to be attainable, even when the cryomodule is on the beam line and cold. Further details of the Brandeis CCD angle monitor (BCAM) alignment system being investigated can be found in [22]. The normalized transverse acceptance of the linac is $2.8 \pi \mathrm{mm} \mathrm{mrad}$ and limited by the aperture of the cavity. In comparison, the maximum possible beam emittance defined by the RFQ acceptance is $0.6 \pi \mathrm{mm}$ mrad. Typically, the emittance of the beam delivered by the REX-EBIS is more than an order of magnitude smaller. A study of the loss of acceptance on the introduction of imperfections in the alignment is summarized in Fig. 19 for a range of different tolerances on the solenoid, BPM $\left(\sigma_{\mathrm{BPM}}\right)$ and cavity $\left(\sigma_{\text {cav }}\right)$. The beam is about 6 times more sensitive to misalignments of the solenoid than the cavity which drove the choice of a separately adjustable support independent of the cavities for the solenoid inside the cryomodule. Only large misalignment of the cavity with respect to the solenoid significantly affects the acceptance. The loss in acceptance depends linearly on the solenoid alignment tolerance and the strength of the relationship depends on the BPM alignment error, which affects the efficacy of the correction of the beam centroid. With an rms alignment tolerance of $\pm 0.38 \mathrm{mrad}( \pm 0.15 \mathrm{~mm}$ ) on the solenoid, $\pm 0.3 \mathrm{~mm}$ on the cavity, and $\pm 0.2 \mathrm{~mm}$ on the BPM, the acceptance is reduced by $25 \%$ and the rms beam core can be kept in the good field region defined by half the radial aperture. This specification demands an integrated field strength of $4 \mathrm{~T}$ $\mathrm{mm}$ from the steerer magnets.

\section{F. Longitudinal error study}

The sensitivity of the beam to the stability of the amplitude and phase in the 20 high- $\beta$ cavities of the intermediate upgrade was investigated using the error module in TRACK. The phase and amplitude errors were parametrized by the 
standard deviation of a Gaussian distribution truncated at $3 \sigma$ and a total of 250 error seeds simulated. In each error seed a bunch containing 2000 particles was tracked. The emittance of the ensemble of bunches representing the time-averaged emittance growth was compared to the case with no amplitude or phase error. The rms emittances of the particle distributions are compared in the jittered and nonjittered cases. The total emittance at injection was simulated as the design value of $2 \pi \mathrm{keV} / \mathrm{u}$ ns.

The emittance grows linearly with the number of independently phased QWRs after the first cavity and the effect of the jitter is very strong in the first cavity because of the large phase spread and low energy of the beam at injection.

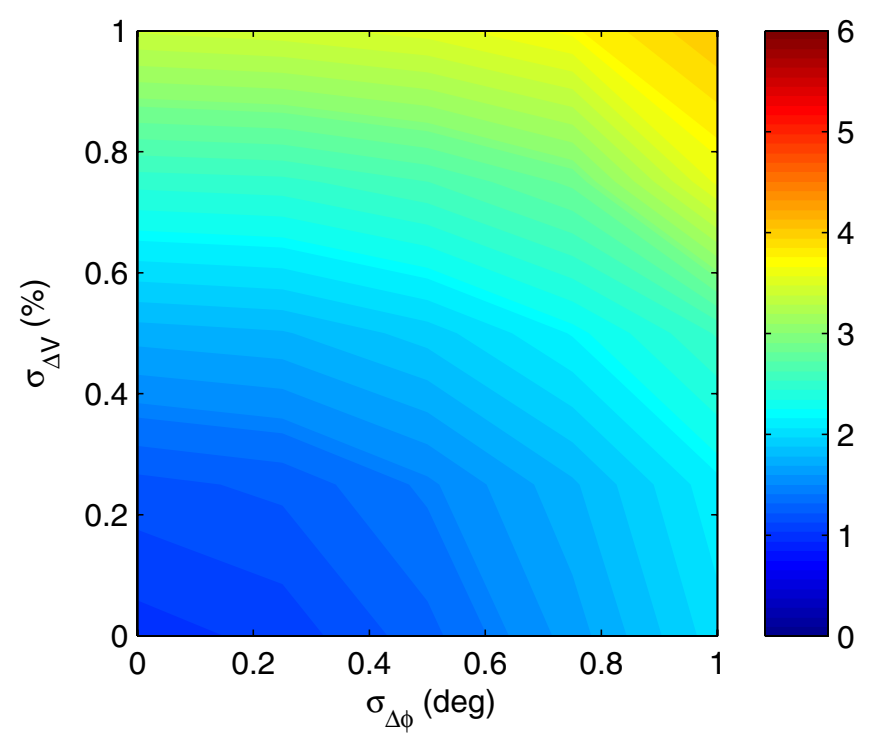

(a) $A / q=4.5$.

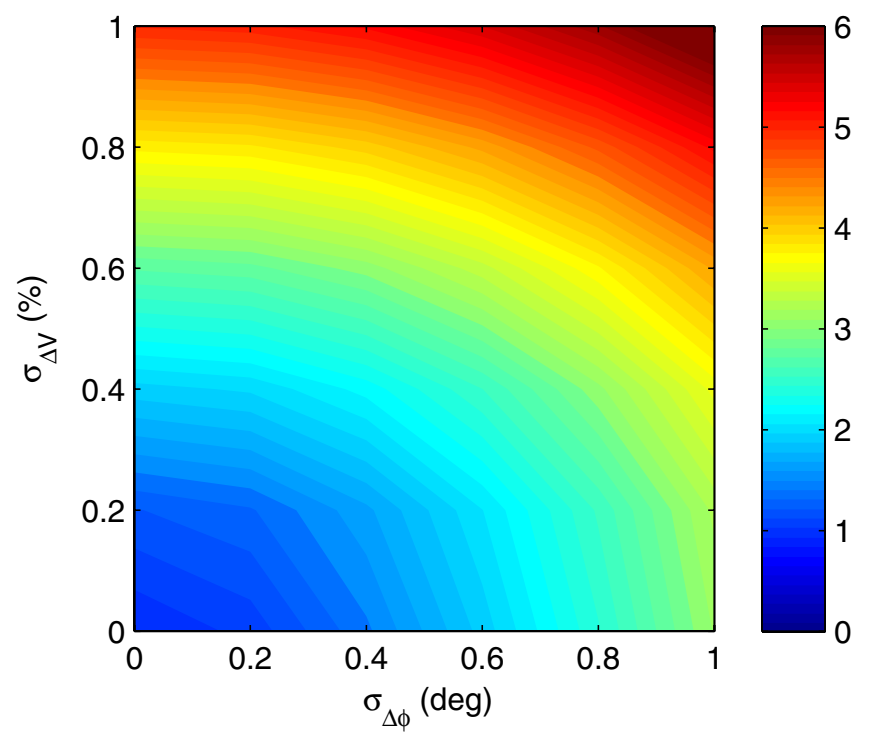

(b) $A / q=2.5$.

FIG. 21. The time-averaged rms longitudinal emittance growth factor as a function of the rf stability in the high energy section.
The results are presented in Fig. 20 for a total jitter in phase and voltage of $1^{\circ}$ and $1 \%$, respectively.

The effect of jitter was surveyed as a function of amplitude and phase error for beams with $A / q=4.5$ and 2.5 accelerated to maximum energy as shown in Fig. 21. The rms longitudinal emittance growth can be kept below 50\% with an rms tolerance of $0.2^{\circ}$ and $0.2 \%$ on the amplitude and phase of each QWR. The energy spread delivered to the experiments can therefore be kept below $0.25 \%$ at $10 \mathrm{MeV} / \mathrm{u}$ for $A / q=4.5$.

\section{SUMMARY}

The specification and design choices for the HIEISOLDE postaccelerator were outlined and the main stages in the beam dynamics study of the high energy section discussed with an emphasis on the mitigation of emittance growth. The strong coupling between the transverse and longitudinal motions encountered as a result of the long focusing periods and high gradient employed to meet the energy gain specification with full flexibility was controlled with a suitably tuned focusing strength. The sources of emittance growth were understood with analytical and numerical models and the design of the QWRs optimized. In addition, the results of error studies were presented to understand the tolerances required to realize the machine.

\section{ACKNOWLEDGMENTS}

The authors would like to thank Bob Laxdal and Peter Ostroumov for helpful discussions during the HIEISOLDE International Review Meeting of 2009 and Alessandro D'Elia for his help in the rf simulations of the high- $\beta$ cavity. Gratitude is also extended to Dmitry Gorelov and Brahim Mustapha for making the implementation of the HIE-ISOLDE linac in the LANA and TRACK codes possible. We would like to acknowledge the support of both Mats Lindroos and Yacine Kadi.

[1] O. Kester et al., in the 14th International Conference on Electromagnetic Isotope Separators and Techniques Related to their Applications [Nucl. Instrum. Methods Phys. Res., Sect. B 204, 20 (2003)].

[2] D. Voulot et al., in Proceedings of the XVth International Conference on Electromagnetic Isotope Separators and Techniques Related to their Applications [Nucl. Instrum. Methods Phys. Res., Sect. B 266, 4103 (2008)].

[3] M. Lindroos et al., in Proceedings of the XVth International Conference on Electromagnetic Isotope Separators and Techniques Related to their Applications [Nucl. Instrum. Methods Phys. Res., Sect. B 266, 4687 (2008)].

[4] A. C. Mueller, R. E. Laxdal, and O. Kester, International Advisory Board Report for the REX-ISOLDE Linac 
Energy Upgrade, Report to AB Department (CERN, 2006), http://www.cern.ch/hie-isolde.

[5] M. Pasini and R.E. Laxdal, TRIUMF Design Note No. TRI-DN-01-08, 2001.

[6] D. V. Gorelov, P. N. Ostroumov, and R. E. Laxdal, in Proceedings of the 1997 Particle Accelerator Conference, Vancouver, BC (IEEE, New York, 1998), pp. 2621-2623.

[7] J. R. Delayen, Nucl. Instrum. Methods Phys. Res., Sect. A 258, 15 (1987).

[8] M. Pasini et al., in Proceedings of the Linear Accelerator Conference, Vancouver, Canada, 2008, pp. 827-829, http://trshare.triumf.ca/ linac08proc/Proceedings/.

[9] A. D'Elia et al., in Proceedings of the Superconducting RF Conference, Berlin, Germany, 2009, pp. 609-613, http:// accelconf.web.cern.ch/AccelConf/srf2009/index.htm.

[10] R. L. Gluckstern, BNL Accelerator Department Internal Report No. AADD-31 (1964).

[11] R. L. Gluckstern, BNL Accelerator Department Internal Report No. AADD-120, 1966.

[12] R. L. Gluckstern, IEEE Trans. Nucl. Sci. 16, 194 (1969).

[13] R. L. Gluckstern, in Linear Accelerators, edited by P. M. Lapostolle and A. L. Septier (North-Holland, Amsterdam, 1970), Chap. 1.2d, pp. 797-804.
[14] M. A. Fraser and M. Pasini, Beam Dynamics Studies of the HIE-LINAC at CERN, HIE-ISOLDE Technical Note (CERN, 2008), http://www.cern.ch/hie-isolde.

[15] P. N. Ostroumov, V. Aseev, and B. Mustapha, TRACK-a Code for Beam Dynamics Simulation in Accelerators and Transport Lines with 3D Electric and Magnetic Fields, ANL, Argonne, IL, USA, v. 37 ed. (2007) (http:// www.phy.anl.gov/atlas/TRACK/).

[16] P. N. Ostroumov and K. W. Shepard, Phys. Rev. ST Accel. Beams 4, 110101 (2001).

[17] A. Facco and V. Zviagintsev, in Proceedings of the Particle Accelerator Conference, Chicago, IL, 2001 (IEEE, New York, 2001), pp. 1095-1097.

[18] B. Carlsten, LANL Report No. AT-7:90-233, 1990.

[19] M. Fraser et al., in Proceedings of the Superconducting RF Conference, Berlin, Germany, 2009, pp. 604-608.

[20] M. A. Fraser and M. Pasini, Misalignment and Error Studies of the High Energy Section of the HIEISOLDE Linac, HIE-ISOLDE Technical Note (CERN, 2009), http://www.cern.ch/hie-isolde.

[21] M. Pasini et al., TRIUMF Design Note No. TRI-DN-0303, 2003.

[22] K. Hashemi, BCAM Home Page, Brandeis University, Waltham, MA, USA (2010), http://alignment.hep .brandeis.edu/Devices/BCAM/. 